\title{
Functional Tissue Engineering: A Prevascularized Cardiac Muscle Construct for Validating Human Mesenchymal Stem Cells Engraftment Potential In Vitro
}

Mani T. Valarmathi, MD, PhD, John W. Fuseler, $\mathrm{PhD}^{2}$, Jay D. Potts, $\mathrm{PhD}$, Jeffrey M. Davis, BS, and Robert L. Price, $\mathrm{PhD}^{3}$

The influence of somatic stem cells in the stimulation of mammalian cardiac muscle regeneration is still in its early stages, and so far, it has been difficult to determine the efficacy of the procedures that have been employed. The outstanding question remains whether stem cells derived from the bone marrow or some other location within or outside of the heart can populate a region of myocardial damage and transform into tissue-specific differentiated progenies, and also exhibit functional synchronization. Consequently, this necessitates the development of an appropriate in vitro threedimensional (3D) model of cardiomyogenesis and prompts the development of a 3D cardiac muscle construct for tissue engineering purposes, especially using the somatic stem cell, human mesenchymal stem cells (hMSCs). To this end, we have created an in vitro 3D functional prevascularized cardiac muscle construct using embryonic cardiac myocytes (eCMs) and hMSCs. First, to generate the prevascularized scaffold, human cardiac microvascular endothelial cells (hCMVECs) and hMSCs were cocultured onto a 3D collagen cell carrier (CCC) for 7 days under vasculogenic culture conditions; hCMVECs/hMSCs underwent maturation, differentiation, and morphogenesis characteristic of microvessels, and formed dense vascular networks. Next, the eCMs and hMSCs were cocultured onto this generated prevascularized CCCs for further 7 or 14 days in myogenic culture conditions. Finally, the vascular and cardiac phenotypic inductions were characterized at the morphological, immunological, biochemical, molecular, and functional levels. Expression and functional analyses of the differentiated progenies revealed neo-cardiomyogenesis and neovasculogenesis. In this milieu, for instance, not only were hMSCs able to couple electromechanically with developing eCMs but were also able to contribute to the developing vasculature as mural cells, respectively. Hence, our unique 3D coculture system provides us a reproducible and quintessential in vitro 3D model of cardiomyogenesis and a functioning prevascularized 3D cardiac graft that can be utilized for personalized medicine.

Keywords: bone marrow stromal cells, cardiovascular tissue engineering, mesenchymal stem cells, embryonic cardiac myocytes, excitation-contraction coupling, myocardial regeneration

\section{Introduction}

T He most FUNDAMENTAL PROBLEM facing cardiac therapy, unlike vascular grafts and heart valves, is to repair and/or regenerate the damaged myocardium. Restricted myocardial regeneration after tissue damage and shortage of donor organs for cardiac transplantation are the major constraints of conventional therapies. ${ }^{1}$ The most daunting task in the field of cardiovascular tissue engineering is the creation and/or regeneration of an in vitro engineered cardiac muscle; tissue engineering is associated with two common underlying concerns for clinical applicability, viz.,

\footnotetext{
${ }^{1}$ Department of Comparative Biosciences, College of Veterinary Medicine, University of Illinois at Urbana-Champaign, Urbana, Illinois. Departments of ${ }^{2}$ Pathology, Microbiology and Immunology and ${ }^{3}$ Cell Biology and Anatomy, School of Medicine, University of South Carolina, Columbia, South Carolina.

(C) Mani T. Valarmathi et al. 2018; Published by Mary Ann Liebert, Inc. This is an Open Access article distributed under the terms of the Creative Commons Attribution License, which permits unrestricted use, distribution, and reproduction in any medium, provided the original work is properly cited.
} 
contractility and thickness. ${ }^{2}$ However, both the thickness and the contractility of the derived cardiac tissue are dependent on the vascularity of the construct.

Until now, no single technique has been proven very effective to generate tissue with all the desirable characteristics of a tissue-engineered cardiac graft: for example, consistent and synchronized contractility, stable electrophysiological properties, vascularization, and most importantly, an autologous cell source. ${ }^{3}$ Thus, strategies aiming to generate a tissue graft using combinatorial approaches to repair a cardiac lesion should be addressed.

Organ tissue engineering, including cardiovascular tissues, has been an area of intense investigation; it aims at replacing and/or regenerating tissues lost due to diseases or trauma. Once again, the major challenge to these approaches has been the inability to vascularize and perfuse the in vitro engineered tissue constructs. ${ }^{4-6}$ Since most in vitro engineered tissue constructs do not contain the intricate microvascular structures resembling those of native tissue, the cells contained in scaffolds, to a large extent, rely on simple diffusion for oxygenation and nutritional delivery. ${ }^{5}$

Mimicking the physiological complexity of a vascularized tissue in vitro is a major obstacle, which would possibly contribute to impaired healing in vivo. ${ }^{7}$ Therefore, engineering a tissue of clinically relevant magnitude requires the formation of an extensive and stable intricate network of microvascular structures within the tissue. Furthermore, scalability is yet another key impediment for successful cardiovascular tissue engineering. ${ }^{8-10}$ Taken together, these facts suggest that, the strategy of prevascularization of tissues is a significant step forward in creating more complex tissues mimicking native tissue physiology and/or physiopathologies. $^{7}$

Both in development and repair, angiogenesis and vascular invasion are a sine qua non for cell differentiation and tissue morphogenesis. Apart from their all-important role in oxygen and nutrient delivery, it has recently been documented that endothelial cells play a crucial role in regulating and maintaining tissue-specific cells, and reported to influence both in early cardiac development and in adult heart. ${ }^{11-13}$

Previous studies pointed out that microvascular endothelial cells promote cardiac myocyte survival and spatial reorganization. ${ }^{11,14,15}$ In addition, in vitro heterotypic primary culture (coculture) of microvascular endothelial cells and ventricular cardiac myocytes has revealed that reciprocal intercellular signaling regulates cardiac growth and function, and operates by means of autocrine and paracrine mechanisms. ${ }^{16}$ Such intercellular signaling has also been shown to regulate cardiac myocyte contractility and apoptosis. ${ }^{17,18}$ In contrast, cardiac myocytes are presumed to influence endothelial cell survival and assembly. In general, these evidences suggest that one of the fruitful strategies for myocardial regeneration may consequently depend on establishing functional myocyte-endothelium communications and/or interactions.

Given these shortcomings and in light of the abovementioned facts, this research work is aimed to address how to develop a three-dimensional (3D) model of vascularized cardiac tissue to study the concurrent temporal and spatial regulation of cardiomyogenesis in the context of postnatal in situ de novo vasculogenesis during stem cell cardiac regeneration. So, we have harnessed the developmental bi- ology principles, the cell-cell interaction and cell-matrix interaction, and tested the following supposition: whether functioning vascularized cardiac tissue can be generated by the simultaneous interaction of cardiac myocytes, endothelial cells, and somatic stem cells, as would be expected to occur during myocardial reparative/regenerative processes, by utilizing, viz., the embryo-derived embryonic cardiac myocytes (eCMs) and the human adipose-derived multipotent mesenchymal stem cells (hMSCs) on a 3D prevascularized collagen cell carrier (CCC) scaffold.

\section{Materials and Methods}

An overview of the modular approach for generating a prevascularized cardiac muscle construct, conceptualized in Supplementary Figure S1 (Supplementary Data are available online at www.liebertpub.com/tea).

\section{eCM culture: plating and maintenance}

All animal procedures were carried out in accordance with the guidelines for animal experimentation set forth and approved by Institutional Animal Care and Use Committee (IACUC), College of Veterinary Medicine, University of Illinois at Urbana-Champaign. eCMs were isolated from E15 timed pregnant Sprague Dawley (SD) rats (Harlan Sprague Dawley, Inc.) as described previously. ${ }^{19,20}$

\section{Human cardiac microvascular endothelial cell culture: plating, maintenance, and subculture}

Human cardiac microvascular endothelial cells (hCMVECs) were purchased from Lonza (Walkersville, MD) and were expanded and subcultured according to the manufacturer's recommendations. Briefly, cells were thawed and seeded onto a fibronectin-precoated T75 flask $\left(1 \mu \mathrm{g} / \mathrm{cm}^{2}\right.$ or $\left.0.5 \mu \mathrm{g} / \mathrm{mL}\right)$ and expanded using a complete microvascular endothelial cell growth medium, supplemented with $5 \%$ fetal bovine serum (FBS), human epidermal growth factor (hEGF), vascular endothelial growth factor (VEGF), R3-insulin-like growth factor1 (R3-IGF-1), human fibroblast growth factor-beta (hFGF- $\beta$ ), ascorbic acid, hydrocortisone, gentamicin, and amphotericin-B (Clonetics $^{\mathrm{TM}} \mathrm{EGM}^{\mathrm{TM}}-2 \mathrm{MV}$ BulletKit ${ }^{\mathrm{TM}}$; Lonza).

\section{hMSC culture: plating, maintenance, and subculture}

hMSCs were obtained from ScienCell Research Laboratories (Carlsbad, CA) and were expanded and maintained as per the manufacturer's instructions. After three passages, the attached hMSCs were trypsinized and subjected to further purification and characterization.

\section{Immunophenotyping of hMSCs by single-color flow cytometry}

Quantitative analysis for various cell surface markers (Table 1) was performed by single-color flow cytometry using a Coulter ${ }^{\circledR}$ EPICS $^{\circledR}$ XL $^{\mathrm{TM}}$ Flow Cytometer as described previously. ${ }^{21,22}$

\section{Enrichment of hMSCs by magnetic-activated cell sorting}

Additional purification and enrichment of the cultured hMSCs were performed as per our previously published method, ${ }^{22}$ using 
Table 1. Primary Antibodies Used in This Study

\begin{tabular}{|c|c|c|c|}
\hline Primary antibodies & Dilutions & Source & Cell target \\
\hline \multicolumn{4}{|l|}{ MSCs characterization markers } \\
\hline$C D 11 B$ & $1: 50$ & BD Pharmingen & Leukocytes \\
\hline$C D 31$ & $1: 10$ & Abcam & Endothelial \\
\hline CD34 & $1: 50$ & Santa Cruz Biotechnology & Hematopoietic \\
\hline$C D 44$ & $1: 10$ & Gene Tex, Inc. & Leukocytes \\
\hline$C D 45$ & $1: 50$ & BD Pharmingen & Leukocytes \\
\hline$C D 73$ & $1: 50$ & BD Pharmingen & $\mathrm{MSCs}$ \\
\hline$C D 90$ & $1: 50$ & BD Pharmingen & $\mathrm{MSCs}$ \\
\hline$C D 106$ & $1: 50$ & BD Pharmingen & Endothelial \\
\hline \multicolumn{4}{|l|}{ Endothelial cell differentiation markers } \\
\hline PECAM1 & $1: 100$ & Santa Cruz Biotechnology & Endothelial \\
\hline$V W F$ & $1: 100$ & Santa Cruz Biotechnology & Endothelial \\
\hline$V E-C A D H E R I N$ & $1: 100$ & Santa Cruz Biotechnology & Endothelial \\
\hline LECTIN & $1: 50$ & Vector Laboratories & Endothelial \\
\hline LAMININ & $1: 200$ & Abcam & Endothelial \\
\hline \multicolumn{4}{|l|}{ Smooth muscle cell differentiation markers } \\
\hline$\alpha-S M A$ & $1: 100$ & Sigma-Aldrich & Smooth muscle \\
\hline CALPONIN & $1: 5000$ & Sigma-Aldrich & Smooth muscle \\
\hline \multicolumn{4}{|l|}{ Cardiac myocyte differentiation markers } \\
\hline Cardiac myosin heavy chain $(\alpha / \beta M y h)$ & $1: 200$ & Abcam & Cardiomyocyte \\
\hline Cardiac troponin $T(c T n T)$ & $1: 200$ & Abcam & Cardiomyocyte \\
\hline Cardiac troponin I (cTnI) & $1: 200$ & Santa Cruz Biotechnology & Cardiomyocyte \\
\hline$\alpha$-Actinin & $1: 200$ & Abcam & Cardiomyocyte \\
\hline$N$-Cadherin & $1: 200$ & Santa Cruz Biotechnology & Cardiomyocyte \\
\hline Connexin $45(C \times 45)$ & $1: 200$ & Santa Cruz Biotechnology & Cardiomyocyte \\
\hline Connexin 43 (Cx43) & $1: 200$ & Santa Cruz Biotechnology & Cardiomyocyte \\
\hline Desmin & $1: 200$ & Abcam & Cardiomyocyte \\
\hline Gata4 & $1: 200$ & Santa Cruz Biotechnology & Cardiomyocyte \\
\hline Bnp & $1: 200$ & Santa Cruz Biotechnology & Cardiomyocyte \\
\hline
\end{tabular}

Bnp, brain natriuretic peptide; Gata4, GATA binding protein 4; PECAM1, platelet and endothelial cell adhesion molecule 1; VWF, von Willebrand factor.

an autoMACS ${ }^{\mathrm{TM}}$ Pro Separator (Miltenyi Biotech). The resulting enriched $C D 45 / C D 34^{-} / C D 90^{+}$fractions were subcultured and expanded further. ${ }^{23}$

\section{Labeling of hMSCs with green fluorescent protein for cell lineage tracing}

Lentiviral vector construction and lentivirus assembly. Lentiviral vector construction and lentivirus production were carried out as per our previously published protocol, ${ }^{20}$ using pWPT-GFP, together with pCMVR8.74 (packaging plasmid) and pMD2.G (envelope plasmid). Finally, the viral titer was ascertained by standard HeLa titer procedure using green fluorescent protein (GFP) as a marker.

hMSC lentiviral transduction. Lentiviral transduction of hMSCs $\left(0.2 \times 10^{6}\right.$ cells/well $)$ using the desired number of viral particles (multiplicity of infection $[\mathrm{MOI}]=5$ ) was performed precisely using the previously published proto$\mathrm{col}^{20}$ and the transduction efficiency was estimated using single-color fluorescence-activated cell sorting (FACS), and was $>95 \%$.

\section{Priming of CCCs for attachment and cell seeding}

CCCs were procured from Viscofan BioEngineering (Weinheim, Germany). ${ }^{24}$ In brief, first, each well of a cell culture-treated 24 -well plate was preloaded with $250 \mu \mathrm{L}$ of prewarmed $\left(37^{\circ} \mathrm{C}\right)$ Dulbecco's phosphate-buffered saline (DPBS), pH 7.4. Next, a sterile CCC was placed on top of the liquid containing wells using a sterile force, avoiding entrapment of air bubbles. Each one of those CCCs was incubated for $30 \mathrm{~min}$ at room temperature. The DPBS was removed and CCCs were washed twice and dried overnight in an operating laminar flow hood with the lid removed or ajar. After overnight drying, the CCCs were attached firmly to the bottom of the well and were ready for cell seeding. Before cell seeding, the CCCs were equilibrated (to reach physiological $\mathrm{pH}$ ) by incubation with an appropriate volume of the desired prewarmed culture medium for at least $30 \mathrm{~min}$ at $37^{\circ} \mathrm{C}$ in the $\mathrm{CO}_{2}$ incubator. Finally, the medium was removed, and the CCCs were subjected to physical crosslinking using Stratalinker ${ }^{\circledR}$ UV Crosslinker 1800 (Stratagene), before seeding cells onto the CCCs.

\section{Generation of prevascularized CCCs}

Seeding of hCMVECs onto CCCs. Next, subcultured and expanded hCMVECs were plated onto the prepared and fibronectin $\left(1 \mu \mathrm{g} / \mathrm{cm}^{2}\right.$ or $\left.0.5 \mu \mathrm{g} / \mathrm{mL}\right)$-precoated CCCs at a density of $0.5 \times 10^{6}$ cells $/ 30 \mathrm{~mm}$ CCC and cultured in a complete microvascular endothelial cell growth medium (Clonetics EGM-2MV BulletKit; Lonza) for either 7 or 14 days. In addition, hCMVECs were grown onto a fibronectin-coated Lab-Tek ${ }^{\mathrm{TM}}$ chamber slide system $^{\mathrm{TM}}$ (Nunc) 
and cultured under similar vasculogenic culture conditions as controls.

Seeding of hMSCs onto CCCs. Similarly, hMSCs were plated onto the prepared and fibronectin-precoated CCCs at a density of $0.3 \times 10^{6}$ cells $/ 30 \mathrm{~mm} \mathrm{CCC}$ and cultured in the complete microvascular endothelial cell growth medium (Clonetics EGM-2MV BulletKit; Lonza) for either 7 or 14 days.

Seeding of hCMVECs and hMSCs onto CCCs. Furthermore, hCMVECs were cocultured with hMSCs $\left(0.5 \times 10^{6}\right.$ of hCMVECs and $0.3 \times 10^{6}$ of $\mathrm{hMSCs} / 30 \mathrm{~mm}$ $\mathrm{CCC}$ ) and cultured in the complete microvascular endothelial cell growth medium (Clonetics EGM-2MV BulletKit; Lonza) for either 7 or 14 days.

The cultures (hCMVECs or hMSCs or hCMVECs/ hMSCs) were terminated at these regular intervals (day 7 or 14), and the collected samples were subjected to reverse transcription-quantitative real-time polymerase chain reaction (RT-qPCR), immunofluorescence, ultrastructural, and biochemical analyses.

\section{Functional characterization of prevascularized CCCs by Dil-conjugated acetylated low-density lipoprotein uptake assay}

Functional characterization of endothelial cells (i.e., CCCs seeded with either hCMVECs or hCMVECs/hMSCs, and cultured under vasculogenic differentiation conditions for 7 or 14 days) was carried out using Dil-conjugated acetylated low-density lipoproteins (Dil-Ac-LDL staining kit; Biomedical Technologies, Inc.), as described previously. ${ }^{25}$ Subsequently, immunostaining of the CCCs with VE-CADHERIN (1:100 dilution) or CALPONIN (1:5000 dilution) was also performed to demarcate either the endothelial or the smooth muscle cells, respectively (as described in detail in Immunofluorescence Staining and Confocal Microscopy section; Table 1). DAPI (4,6-diamidino-2-phenylindole, $100 \mathrm{ng} / \mathrm{mL}$; Sigma-Aldrich) was used to counterstain the nuclei. The images of the stained cells were captured using an Olympus BX53 fluorescence microscope system.

\section{Quantification of angiogenesis in prevascularized CCCs}

Analysis of various parameters characterizing the formation of endothelial cell microvascular networks in day 14 vasculogenic hCMVEC CCC cultures and hCMVEC/hMSC cocultures, such as junctions (group of joined nodes, pixels with at least three neighbors), branches (elements delimited by a junction and one extremity), isolated segments (binary lines that are not branched), meshes (areas enclosed by segments), total length of endothelial network (interconnected segments, branches, and isolated segments), and percentage $(\%)$ branching length (length of interconnected segments and branches divided by total network length), was performed by employing ImageJ software ${ }^{26}$ (Angiogenesis Analyzer, an ImageJ plugin). ${ }^{27,28}$

In addition, quantitative analysis of various parameters typifying the generation of both muscular microvessels (>20-130 $\mu \mathrm{m}$ wide) and muscular macrovessels $(>130 \mu \mathrm{m}$ wide), such as vessel length and breadth, in day 14 vasculogenic hCMVEC/hMSC CCC cocultures using the ImageJ software, ${ }^{26}$ and likewise, areas covered by endothelial and smooth muscle cells of both small-caliber $(<20 \mu \mathrm{m}$ wide $)$ and large-caliber vessels ( $>20 \mu \mathrm{m}$ wide), in day 14 vasculogenic hCMVEC/hMSC CCC cocultures, were analyzed.

\section{Scanning electron microscopic analysis of prevascularized CCCs}

To depict the nature and structural organization of the vascular component, the day 14 CCC samples (i.e., hCMVEC/ hMSC CCCs cocultures under vasculogenic culture conditions) were processed for scanning electron microscopy (SEM) by means of the O-GTA-O-GTA-O method. ${ }^{29}$

\section{Generation of avascularized cardiac graft}

Seeding eCMs onto CCCs. The isolated embryonic ventricular primary cells, eCMs, were quantified using a hemocytometer and plated at a density of $2 \times 10^{5}$ cells/well of a 24-well bottom culture dish that was preattached with individual CCCs, incubated in a humidified atmosphere of $5 \% \mathrm{CO}_{2}$ at $37^{\circ} \mathrm{C}$ for $48 \mathrm{~h}$, and observed under inverted phase contrast microscope (Olympus IX73) for spontaneous beating and rhythmic contractions. These eCMs were cultured in a complete myocyte growth medium (Dulbecco's Modified Eagle Medium [DMEM] with $8 \%$ horse serum [HS] and 5\% newborn calf serum [NCS]) for further 7 or 14 days.

Seeding hMSCs onto CCCs. In addition, the purified and enriched population of hMSCs $\left(C D 90^{+}\right)$was plated onto CCCs at a density of $0.4 \times 10^{5}$ cells/CCC, and cultured in the complete myocyte medium for further 7 or 14 days.

Seeding eCMs and hMSCs onto CCCs. Similarly, the purified and enriched population of hMSCs $\left(C D 90^{+}\right)$(GFP unlabeled or GFP labeled) was plated onto the surface of the previously produced eCMs $\left(2 \times 10^{5}\right.$ cells $)$ containing CCC's wells (after $48 \mathrm{~h}$ ) at a density of $0.4 \times 10^{5}$ cells/well, and were cultured in the complete myocyte growth medium for 7 or 14 days.

The cultures (eCMs or hMSCs or eCM/hMSC-GFP unlabeled or eCM/hMSC-GFP labeled) were terminated at these regular intervals (day 7 or 14), and the collected samples were subjected to RT-qPCR, immunofluorescence, ultrastructural, calcium transit, as well as pharmacological analyses.

\section{Creation of prevascularized cardiac muscle construct-cardiac graft}

Seeding eCMs and hMSCs onto prevascularized CCCs. The eCMs $\left(2 \times 10^{5}\right.$ cells/well $)$ and GFP-unlabeled hMSCs $\left(0.4 \times 10^{5}\right.$ cells/well $)$ were simultaneously added on top of prevascularized CCCs that were cultured in a vasculogenic medium for 7 days, that is, CCCs that were created by the combination coculture of hCMVECs/hMSCs and cultured further in the complete myogenic medium for 7 or 14 days.

Finally, the prevascularized cardiac CCCs (cardiac graft), now containing all three categories of cells, viz., hCMVECs, eCMs, and GFP-unlabeled hMSCs, were terminated at these regular intervals (day 7 or 14), and the collected samples were subjected to immunofluorescence staining and confocal microscopic analysis to validate the simultaneous presence of both vascular and muscular components in these CCC cardiac grafts. 


\section{eCM and hMSC CCC contractility and calcium flux assays}

Loading of cells with Calcium Orange or Fluo-4 calcium indicators. Loading of codifferentiating cells (CCC cultures-eCMs or hMSC-GFP labeled or eCM/hMSC-GFP labeled) with calcium indicator, either Calcium Orange or Fluo-4, was carried out as described previously. ${ }^{20}$ Live-cell imaging using the spinning disk confocal microscopy was employed to record the changes in the intracellular $\mathrm{Ca}^{2+}$ flux of these calcium indicator-labeled cells.

Live-cell imaging using spinning disk confocal microscope. Ultimately, changes in intracellular $\mathrm{Ca}^{2+}$ flux (all types of cells in the CCCs) were examined as elaborated previously, ${ }^{20}$ and by using the AQM Advance- 6 software.

\section{Transmission electron microscopic analysis of CCCs}

To elucidate the ultrastructural characteristics of codifferentiating cells, day 14 CCC samples (i.e., CCCs that were seeded with cells: hMSCs or eCMs, or hMSCs/eCMs or hCMVECs/hMSCs, and cultured using an appropriate medium) were processed for transmission electron microscopic (TEM) analysis as described elsewhere. ${ }^{23}$

\section{Reverse transcription-quantitative real-time polymerase chain reaction}

Total cellular RNA isolation from three independent CCC cultures of various combinations (vasculogenic: hCMVEC culture and hCMVEC/hMSC coculture; cardiomyogenic: eCM culture and eCM/hMSC coculture) that were maintained either in the vasculogenic medium or the myogenic medium were performed using the TRIzol ${ }^{\circledR}$ Plus RNA purification kit (Invitrogen) as per manufacturer's instructions.

The RNA integrity (RIN) of the extracted samples was analyzed on the Agilent 2100 Bioanalyzer system using the Agilent RNA 6000 nano kit (Agilent Technologies, Inc.) following the manufacturer's recommendations. The RT reaction was executed using $250 \mathrm{ng}$ of total RNA in a final reaction volume of $20 \mu \mathrm{L}$ using an iScript ${ }^{\mathrm{TM}}$ Reverse Transcription Supermix for the RT-qPCR kit (Bio-Rad Laboratories, Inc.) according to the manufacturer's protocols.

The cardiomyogenic gene-specific primers for Myh6 (myosin heavy chain 6), Myh7 (myosin heavy chain 7), Actc1 (actin, alpha, cardiac muscle 1), Tnni3 (troponin I3, cardiac type), Gata4 (GATA binding protein 4), Nppa (natriuretic peptide A), Nppb (natriuretic peptide B), and Gjal (gap junction protein, alpha 1), and the vasculogenic geneassociated primers for PECAM1 (platelet and endothelial cell adhesion molecule 1), KDR (kinase insert domain receptor, a type III receptor tyrosine kinase), TIE1 (tyrosine kinase with immunoglobulin-like and EGF-like domains 1), TEK (TEK tyrosine kinase, endothelial), and VWF (von Willebrand factor), as well as the endogenous normalizer reference genes (rat or human) Gapdh/GAPDH (glyceraldehyde-3phosphate dehydrogenase), Actb/ $\beta$-actin (Actin, beta), G6pd/ G6PD (glucose-6-phosphate dehydrogenase), and RplpO/ $R P L P O$ (ribosomal protein lateral stalk subunit P0) were designed using web-based software Primer $3,{ }^{30}$ synthesized commercially (Integrated DNA Technologies, Inc.), and eval- uated for a uniform annealing temperature of $58^{\circ} \mathrm{C}$, for all the primer pairs, as shown in Table 2 .

Real-time PCR conditions were optimized as described previously. $^{21,31-33}$ All RT-qPCRs were performed with SsoAdvanced $^{\mathrm{TM}}$ SYBR $^{\circledR}$ Green Supermix in a CFX96 Touch $^{\text {TM }}$ Real-Time PCR Detection System (Bio-Rad Laboratories, Inc.) and $\mathrm{C}_{\mathrm{T}}$ (threshold cycle) values were calculated using the CFX Manager ${ }^{\mathrm{TM}}$ software, Security Edition. The calibrator control included hCMVEC day 0 sample for vasculogenic cultures and eCM day 0 sample for cardiomyogenic cultures, and the target gene expression was normalized by a set of three nonregulated reference gene expressions, viz., Gapdh/GAPDH, Actb/ $\beta$-actin, and either G6pd/G6PD or Rplp0/RPLPO. The expression ratio of genes was determined by applying the mathematical model previously described by Pfaffl et al. ${ }^{34}$

\section{Immunofluorescence staining and confocal microscopy}

CCC culture samples (vasculogenic: hCMVEC culture, hMSC culture, hCMVEC/hMSC coculture; cardiomyogenic: eCM culture; vascularized cardiac graft: hCMVEC/eCM/ hMSC tri-cell coculture) were collected on day 7 or 14 , and processed according to previously described protocols, ${ }^{20}$ for immunostaining and phalloidin staining. The primary antibodies that were used in this study are shown in Table 1. Rhodamine-labeled Ulex Europaeus Agglutinin I (1:50 in $10 \mathrm{mM} \mathrm{N}$-2-hydroxyethylpiperazine-n'-2-ethanesulfonic acid, pH 7.5; 0.15 M NaCl; LECTIN; Vector Labs) was also used to detect endothelial cells. DAPI $(100 \mathrm{ng} / \mathrm{mL}$; SigmaAldrich) was used to counterstain the nuclei. Images of the stained CCCs were visualized using a confocal (Zeiss LSM 510 Meta CSLM) or a fluorescence (Olympus BX53) microscopic system. Negative controls for staining included only secondary antibodies.

\section{Pharmacological assay of vascularized cardiac graft by live-cell imaging with spinning disk confocal microscopy}

To assess the in vitro functional competence of the generated prevascularized cardiac graft, CCC constructs were exposed to various cardioactive pharmacological agents $(0.1-$ $1 \mu \mathrm{M})$, such as isoprenaline (IPN), clenbuterol (CLEN), and diltiazem (DTZ), either individually or in sequential combination. Calcium oscillations were recorded by live-cell imaging using spinning disk confocal microscopy as described above, and the cell's chronotropic and ionotropic responses were recorded and analyzed.

\section{Statistical analysis}

The RT-qPCR experimental data were represented as mean \pm standard error of the mean. The differences in expression profile (cardiomyogenic and vasculogenic markers) between control (day 0) and treated samples (day 7 or 14) were determined in-group means for statistical significance by applying "Pair Wise Fixed Reallocation Randomization Test" using Relative Expression Software Tool-384 (REST$384^{\complement}$, version 2). ${ }^{34}$ Likewise, other acquired quantitative data, such as image (morphometric and angiogenic) analysis and pharmacological assay, were represented as mean \pm standard deviation of the mean (mean \pm SDM). The differences among 


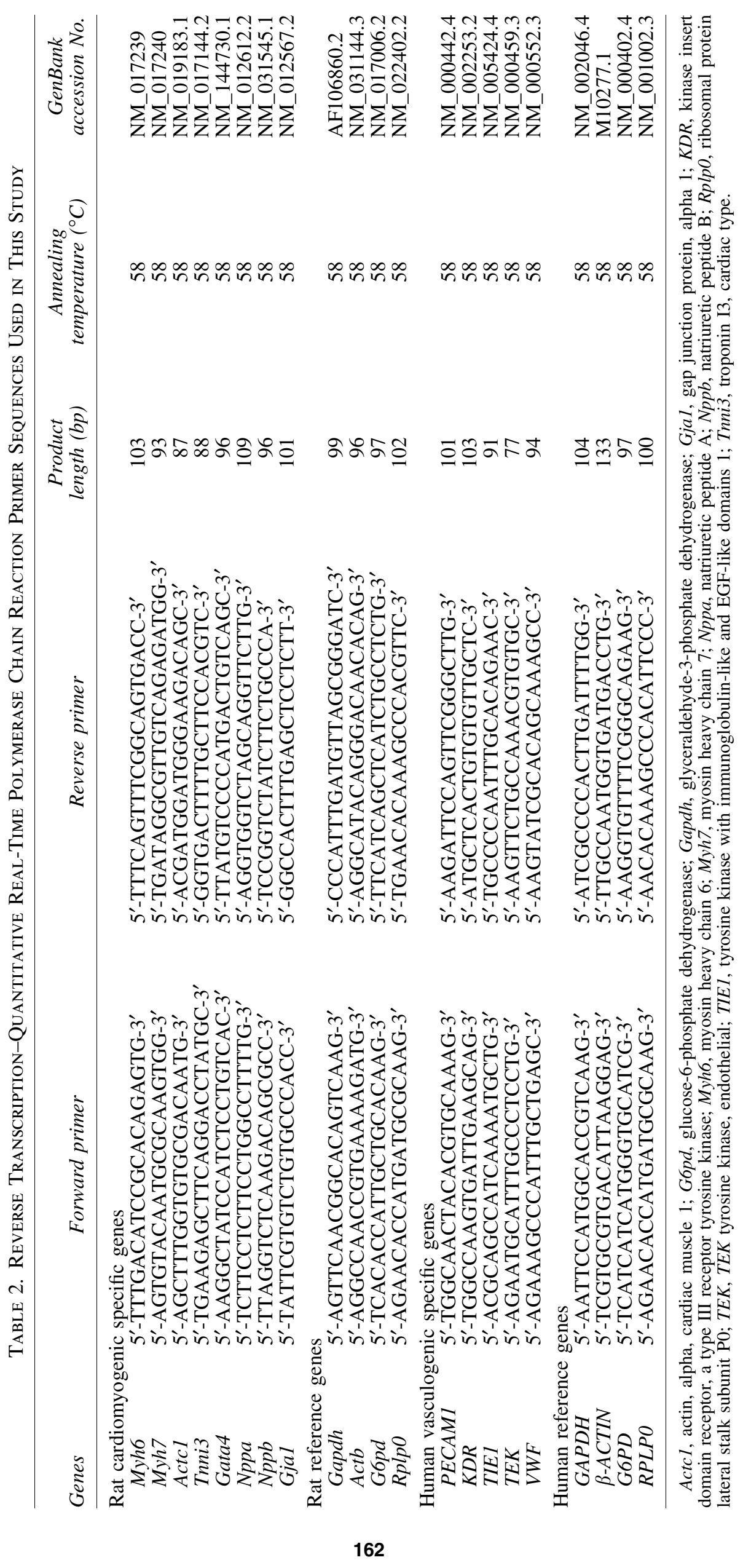


various groups were determined by applying "one-way ANOVA and/or Student's $t$-test." In the case of microscopic analyses, the number of replicates refers to the number of analyzed images, whereas, in the case of pharmacological analyses, the number of replicates refers to the number of analyzed cells. In all cases, $p$-values $<0.05$ were considered statistically significant.

\section{Results}

Immunophenotyping of undifferentiated adipose-derived hMSCs

Since hMSCs are clonogenic in origin and are fundamentally a heterogeneous population of cells, we have analyzed the hMSCs for various significant cell surface markers, before they could possibly be used in all of our experimental setups. Analysis of positive and negative cell surface antigen expression of a pool of expanded (passage 3 maintained) human adipose-derived MSCs by single-color flow cytometry validated that the fluorescent intensity and distribution of the cells examined for angiohematopoietic cell-surface markers, such as $C D 11 B(0.13 \%), C D 31$ (0.30\%), CD34 (0.09\%), CD44 (0.17\%), CD45 (0.13\%), and $C D 106$ (3.19\%), were not significantly different from that of isotype controls (Supplementary Fig. S2A-E, H), whereas on examination, hMSCs displayed a high level expression of MSC-associated surface antigens, such as CD73 (92.22\%) and $C D 90$ (99.66\%) (Supplementary Fig. S2F, G).

These results indicated that the passage 3-pooled population of cells contained almost pure population of MSCs, and the cultures were devoid of any hematopoietic stem and/ or progenitor cells as well as matured endothelial cells. ${ }^{35-37}$

\section{Immunolocalization of vascular lineage-specific markers in prevascularized CCCs}

Next, using modular approach, we have created the prevascularized CCCs. hCMVECs alone, when plated onto fibronectin-precoated CCCs and grown for 14 days under vasculogenic culture conditions produced restricted number of capillary plexuses. Immunolocalization displayed not only areas of flattened and cohesive polyhedral type of cells but also revealed evolving nascent capillary structures with its associated luminal cavity (white arrow, Fig. 1A, B). These structures were strongly positive for a set of endotheliumassociated markers, viz., the PECAM1, VE-CADHERIN, VWF, and LECTIN (Fig. 1A-D). These results indicated that the scaffold, CCCs, were in fact able to provide optimal substratum for the hCMVECs to undergo cellular adhesion, proliferation, differentiation, and morphogenesis.

However, hCMVECs, which were grown on fibronectinprecoated Lab-Tek chamber slide system under identical culture conditions, showed the expression pattern of PECAM1, LECTIN, and VWF (Fig. 1E, F), and the morphological characteristics of polygonal type of cells with conspicuous intercellular bridges, that is, the slender cytoplasmic strands connecting adjacent cells (Fig. 1E, F).

In sharp contrast, when hCMVECs/hMSCs were plated together onto fibronectin-precoated CCCs and grown for 14 days under vasculogenic culture conditions, they dramatically generated dense network of microvessels. Immunolocalization of vasculogenic differentiation antigens demonstrated that the branching plexuses of microvessels were not only positive for key endothelial cell phenotypic markers but also positive for key smooth muscle cell markers, for example, VE-CADHERIN, PECAM1, VWF, $\alpha-S M A$, and LAMININ (Fig. 2A-F). The endothelial cells were organized into alternate layers of flattened cohesive polyhedral cells (bottom layer, Fig. 2A and Supplementary Video S1), and elongated and thinned-out cells assembled into linear and interconnected capillaries (top layer, Fig. 2B), indicative of cellular adhesion, migration, and endothelial tube formation. It was evident that there existed a continuum of transition between these two morphological layers (Fig. 2C), a sign of progressive vascular morphogenesis (Fig. 2A-C and Supplementary Videos S2 and S3). Thus, capillaries were present at various stages of maturation and differentiation, that is, composed of not only varying degrees of matured endothelial and parallelly arranged smooth muscle cells but also, the linear and branching vascular structures were covered with LAMININ molecules, evoking the formation of an intact basement membrane (Fig. 2D-F and Supplementary Video S4).

On the other hand, hMSCs alone grown on fibronectinprecoated CCCs under similar vasculogenic culture conditions assumed elongated and/or stellate type of cellular configuration, and expressed the early- and late-stage smooth muscle cell phenotypic markers, such as $\alpha-S M A$ and CALPONIN, respectively (Fig. 2G, H). These cells were uniformly negative for the endothelial cell markers.

In addition, the hCMVEC/hMSC day 14 cocultures revealed large-caliber vessels, such as emerging mediumsized muscular vessels (Fig. 3A-H). Immunolocalization of vascular differentiation antigens explicitly revealed the morphogenesis of these medium-sized muscular vessels in snapshots. The $V W F$-positive endothelial cells remodeled into a linear broad array of cellular structures and were surrounded by the migrating hMSC-derived $\alpha$-SMA-positive cells (Fig. 3A, B). These mural cells were oriented in a direction that was, in fact, perpendicular to the direction of the longitudinally reassembled endothelial cells (Fig. 3B, C). Further morphogenesis captured the evolving dynamic process of vessel maturation, that is, smooth muscle cells migrated toward the endothelium-lined tubular structures, ultimately wrapping around these tubular structures in a concentric manner (Fig. 3D).

Further stages of maturation and differentiation displayed emergence of large-caliber vessels, showing outer sleevelike mural cells encasing the LAMININ-positive endotheliumlined tubular structures (Fig. 3E-H and Supplementary Videos S5 and S6), reminiscent of mature vascular phenotype. Thus, these snapshots may recapitulate several aspects of in vivo morphogenesis of medium- to large-sized muscular vessels.

\section{Functional characterization of prevascularized CCC construct}

The phenotypic and functional nature of the preformed vessels were validated by means of a classical low-density lipoprotein (LDL) uptake assay. Fluorescent microscopic analysis of Dil-Ac-LDL-stained prevascularized CCC constructs (hCMVEC cultures and hCMVEC/hMSC cocultures) revealed intense uptake of LDL from the culture medium. In 

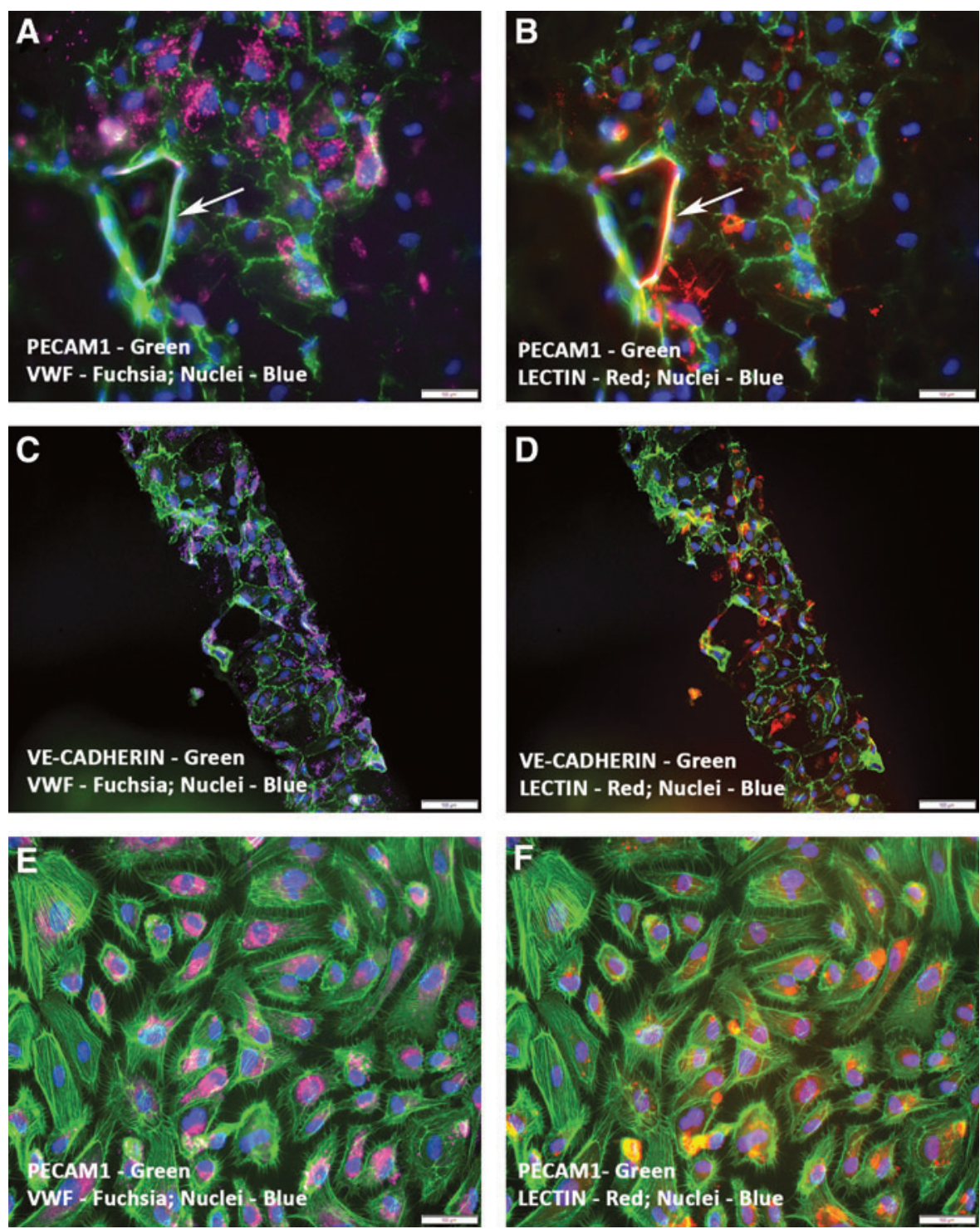

FIG. 1. Expression pattern of key vasculogenic markers in CCCs by immunofluorescence and confocal microscopyhCMVEC culture. Immunostaining of day 14 hCMVEC CCC cultures revealed the expression pattern of PECAM1 (A, B), $V E$-CADHERIN $(\mathbf{C}, \mathbf{D}), \operatorname{LECTIN}(\mathbf{B}, \mathbf{D})$, and $V W F(\mathbf{A}, \mathbf{C})$. Detection of key endothelial cell phenotypic markers illustrated areas of flattened polyhedral cells, and were arranged in a cobble-stone appearance (A-D). Amidst these adherent sheets of polyhedral type of cells were seen nascent capillary structures with its central lumens (white arrow, A, B). In other areas of CCCs, it was evident that these adherent populations of flattened cells were able to retract from the substratum, and remodeled or self-organized into a column of flattened cells $(\mathbf{C}, \mathbf{D})$. These reoriented and evolving aligned broad band of flattened cells were reminiscent of an earlier event of vasculogenesis $(\mathbf{C}, \mathbf{D})$. In contrast, hCMVECs grown on Lab-Tek chamber slide system $^{\mathrm{TM}}$ showed the expression pattern of PECAM1 (E, F), LECTIN (F), and VWF (E), and the morphological characteristics of polygonal type of cells, note the prominent intercellular bridges, slender cytoplasmic strands connecting adjacent cells (E, F). Cells were also counterstained for nuclei (blue, DAPI). Merged images (A-F). (A-F, scale bar $100 \mu \mathrm{m})$. CCCs, collagen cell carriers; DAPI, 4,6-diamidino-2-phenylindole; hCMVECs, human cardiac microvascular endothelial cells; PECAM1, platelet and endothelial cell adhesion molecule 1; VWF, von Willebrand factor. Fig. 1A-D, reproduced with permission from Valarmathi et al., $2017^{37}$.

hCMVEC cultures, the functionally competent and Dillabeled (fluorescent probe) endothelial cells were organized into a dense network of capillaries (Fig. 4A), as well as cohesive array of solid cord of $V E$-CADHERIN-positive cells (Fig. 4B), and were strongly positive for the Dil staining, demonstrating that the endothelial cells were able to internalize or endocytose the Dil-Ac-LDL.
In hCMVEC/hMSC CCCs, the metabolically active endothelial cells showed profuse punctate perinuclear red fluorescence and were able to be distinguished from other types of differentiating cells, such as hMSC-derived smooth muscle cells, especially by their lack of detectable red fluorescence signal (Fig. 4C, D). Figure 4C and D show a typical bright red-stained endothelium-lined tubular vessel, 

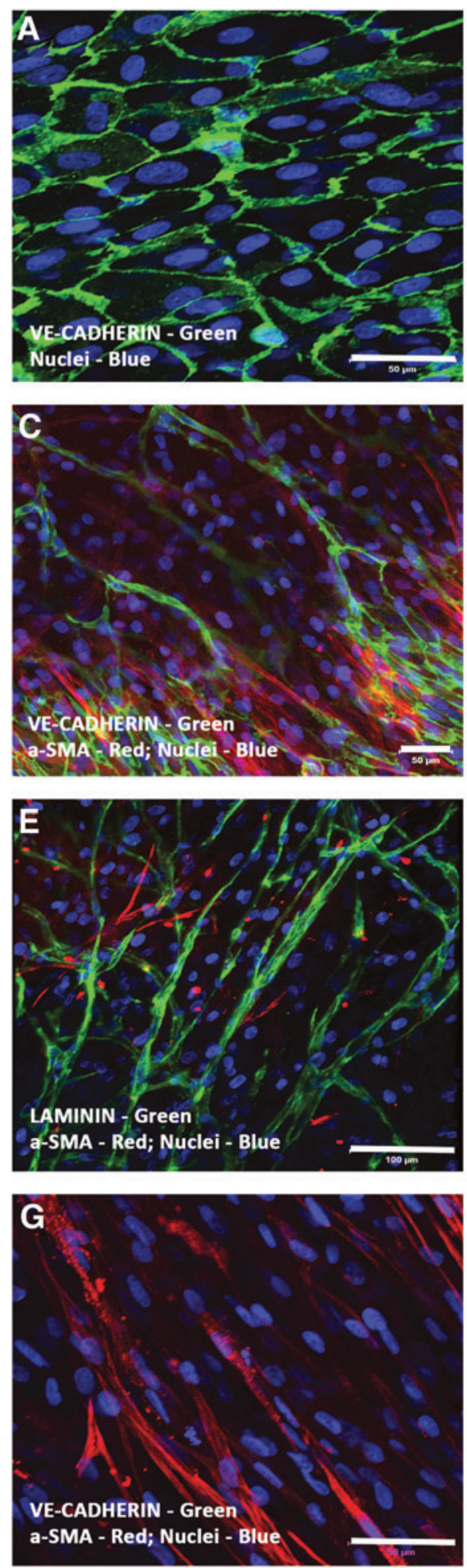
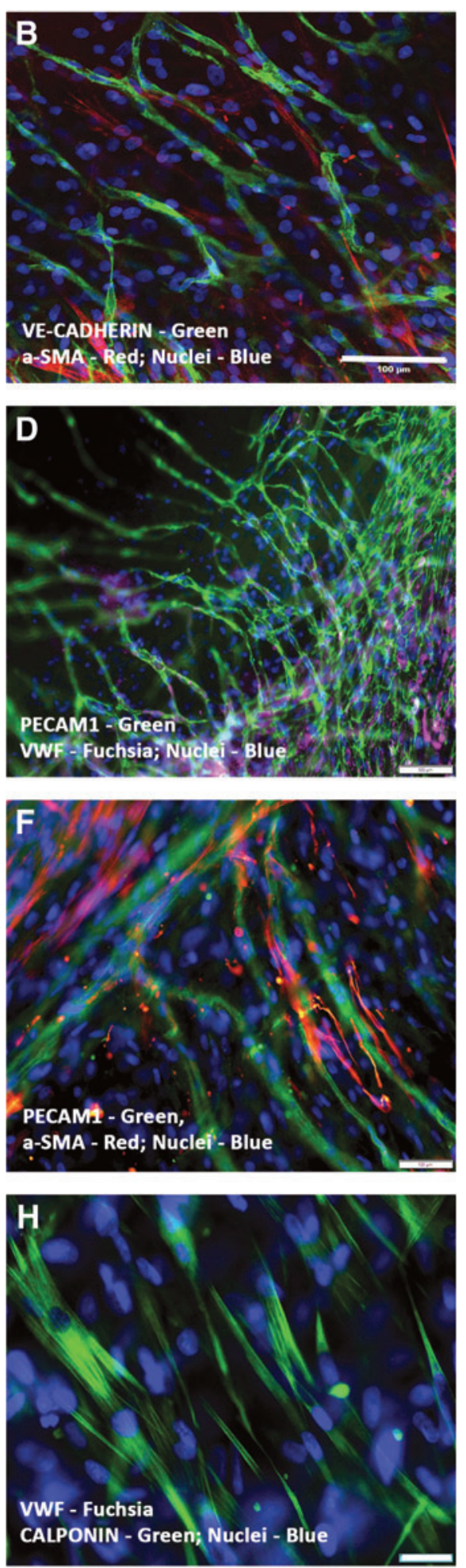

FIG. 2. Expression pattern of key vasculogenic markers in CCCs by immunofluorescence and confocal microscopy-hCMVEC/hMSC coculture. Immunostaining of day 14 hCMVEC/hMSC CCC cocultures revealed the expression pattern of VE-CADHERIN (A-C), PECAMI (D, F), LAMININ (E), VWF (D), and $\alpha-S M A(\mathbf{B}, \mathbf{C}, \mathbf{E}, \mathbf{F})$. Detection of key endothelial cell phenotypic markers illustrated alternating layers of interior flattened cells (bottom layer, A) and exterior elongated cells (top layer, B), indicative of progressive vascular morphogenesis $(\mathbf{C})$, that is, the continuum of the process of vasculogenesis (A-C). Capillaries were present at various stages of maturation, that is, composed of not only varying degrees of matured endothelial and smooth muscle cells, but also dense network of microvessels, which displayed the deposition of LAMININ molecules as the capillary tubes formed, mimicking the formation of an intact basement membrane (E). These developing vascular structures, at higher magnifications, revealed a central slit-like translucent lumen. In contrast, day $14 \mathrm{hMSC}$ CCC cultures under similar culture conditions displayed elongated and/or stellate type of cells, and expressed smooth muscle cell phenotypic markers, such as $\alpha$ SMA and CALPONIN, respectively $(\mathbf{G}, \mathbf{H})$. These cells were consistently negative for the endothelial cell phenotypic markers $(\mathbf{G}, \mathbf{H})$. Cells were also counterstained for nuclei (blue, DAPI). Merged images (A-H). (A, C, G, scale bar $50 \mu \mathrm{m} ; \mathbf{B}, \mathbf{D}, \mathbf{E}, \mathbf{F}, \mathbf{H}$, scale bar $100 \mu \mathrm{m})$. hMSCs, human mesenchymal stem cells. evoking a functionally and metabolically competent largecaliber vessel (Fig. 4C, D).

\section{Analysis of angiogenesis in prevascularized CCCs by ImageJ}

Comparative quantitative analysis of various parameters depicting the development of endothelial cell microvascular networks (Fig. 5A-F and Supplementary Fig. S3A-F), revealed that the measured values, viz., total length $(p=0.001)$, number of meshes $(p=0.001)$, and number of junctions $(p=0.001)$, of day 14 vasculogenic hCMVEC/ hMSC CCC cocultures were significantly greater compared with day 14 vasculogenic hCMVEC cultures (Fig. 5A, D, E). However, there were no significant differences between these two culture groups with respect to the other measured parameters, viz., percentage $(\%)$ branching of total length $(p=0.129)$, number of branches $(p=0.143)$, and number of 
FIG. 3. Characterization of largecaliber muscular vessels in CCCs by immunofluorescence and confocal microscopy-hCMVEC/ hMSC coculture. hCMVECs/ hMSCs day 14 CCC cocultures produced not only dense networks of endothelium-lined capillary plexuses but also vascular structures resembling that of largecaliber muscular vessels (A-H). The plexuses of flattened as well as thin and elongated cells expressed $V W F$-positive endothelial cells and $\alpha$-SMA-positive smooth muscle cells (A-D). The $V W F$-positive endothelial cells remodeled into linear, broad solid cord-like structures, and were uniformly surrounded by the migrating $\alpha-S M A-$ positive mural cells (B). These mural cells were oriented in a direction that was indeed perpendicular to the direction of the longitudinally reassembled endothelial cells (B-D). Further morphogenesis illustrated the evolving dynamic process of vessel maturation, that is, smooth muscle cells migrated toward the endotheliumlined tubular structures (E), eventually wrapping around the entire circumference of these LAMININpositive endothelial outgrowths (FG). Ensuing stages of morphogenesis revealed emergence of largecaliber vessels, showing outer sleeve-like mural cells enclosing the endothelium-lined tubular structures $\mathbf{( H )}$. Cells were also counterstained for nuclei (blue, DAPI). Merged images (A-H). (F, G, scale bar $50 \mu \mathrm{m} ; \mathbf{A}-\mathbf{E}$, $\mathbf{H}$, scale bar $100 \mu \mathrm{m})$.
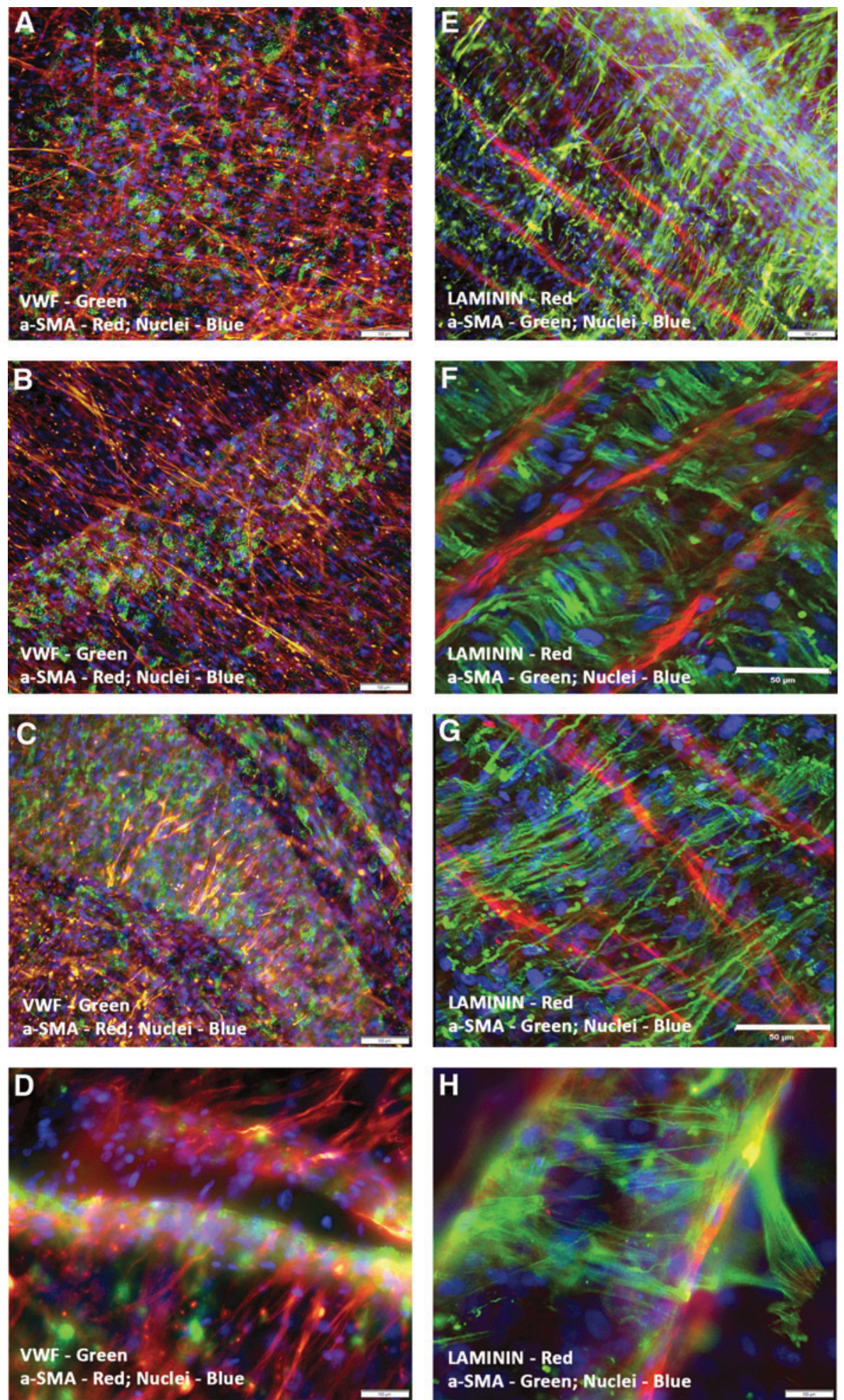

isolated segments $(p=0.133)$ (Fig. 5B, C, F). The results would suggest that the mere presence of hMSCs, in fact, enhanced the angiogenic potential of hCMVECs in this milieu, and could possibly be attributed to the proangiogenic characteristics of hMSCs.

Furthermore, quantitative analysis of a couple of other parameters typifying the formation of large-caliber vessels in day 14 vasculogenic hCMVEC/hMSC cocultures (Fig. 6A,
B) showed that the measured values, such as vessel breadth $(p<0.0001)$ of day 14 muscular macrovessels, were significantly higher compared with day 14 muscular microvessels (Fig. 6B). Nevertheless, no significant difference was observed between these two groups of muscular vessels with respect to the other measured parameter, viz., vessel length $(p=0.740)$ (Fig. 6A). Similarly, no statistically significant differences were observed between the area covered by 

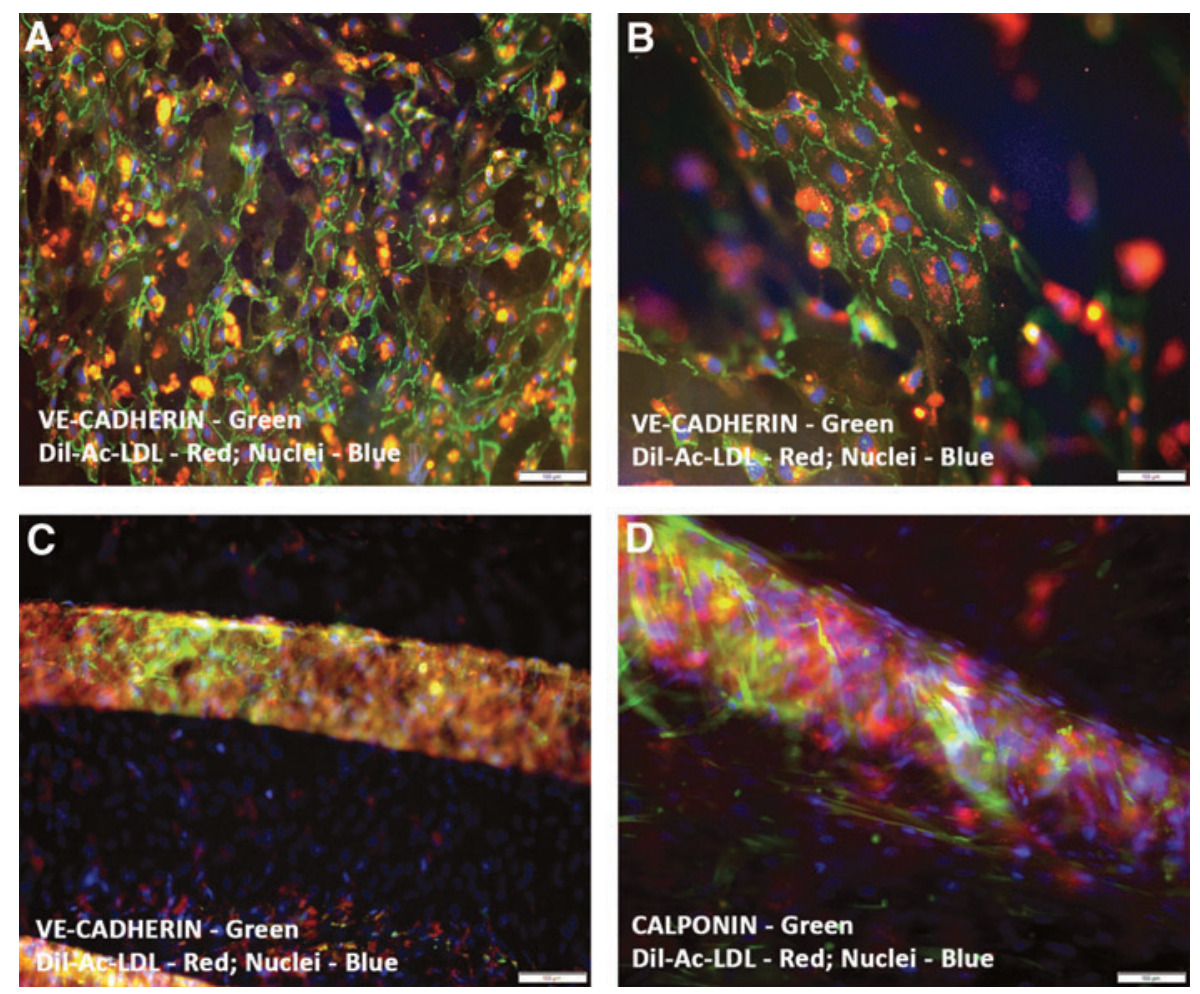

FIG. 4. Functional characterization of prevascularized CCCs by Dil-Ac-LDL uptake assay-hCMVEC culture and hCMVEC/hMSC coculture. Fluorescent microscopic analysis of day 14 hCMVEC CCC cultures labeled with Dil-Ac-LDL identified the endothelial cells that have internalized or endocytosed the Dil-Ac-LDL. These endothelial cells showed profuse punctate perinuclear red fluorescence in their cytoplasm (A, B). The functionally competent and the Dil-labeled (fluorescent probe) endothelial cells were organized into a dense network of capillaries (A), as well as broad band of selforganized cells (B). Likewise, fluorescent microscopic analysis of day $14 \mathrm{hCMVEC/hMSC} \mathrm{CCC} \mathrm{cocultures} \mathrm{revealed}$ intracellular accumulation of Dil (C, D). In addition, these Dil-labeled and metabolically active cells were self-assembled into a cohesive solid cord of VE-CADHERIN-positive cells, evoking a functionally and metabolically competent largecaliber vessel (C), and were able to be discriminated from other types of surrounding differentiated cells such as hMSCderived CALPONIN-positive smooth muscle cells (D). Cells were also counterstained for nuclei (blue, DAPI), endothelial cells (green, VE-CADHERIN, A-C), and smooth muscle cells (green, CALPONIN, D). Merged images (A-D), (A-D, scale bar $100 \mu \mathrm{m})$. Dil-Ac-LDL, Dil-conjugated acetylated low-density lipoprotein.

endothelial cells and the area covered by smooth muscle cells in muscular microvessels $(p=0.115)$, in muscular macrovessels $(p=0.073)$, and in microvascular networks $(p=0.764)$ (Fig. 6C-E). These results would signify that reciprocal induction and differentiation of hCMVECs and hMSCs could support and eventually promote the generation of large-caliber vessels in this microenvironmental niche.

\section{SEM analysis of prevascularized CCCs}

SEM examination of day 14 hCMVEC/hMSC CCC cocultures under vasculogenic culture conditions depicted a typical capillary-like structure with its central cavity (white asterisk, Fig. 7A). The transversely oriented capillaries were lined by flattened layer of one to three differentiating cells. In addition, these cells revealed foci of multilayered cellular aggregation, suggesting cellular retraction from the underlying substratum and remodeling (Fig. 7B). Apart from this, there were prominent linear and convoluted interconnected tubular structures with its encircling smooth muscle cells, that is, the mural type of cells. Some of these cylindrical and/or cord-like structures revealed the emerging hollowing or tunnel-like spaces (white asterisk, Fig. 7C, D).

\section{TEM analysis of prevascularized CCCs}

TEM analysis of day $14 \mathrm{hCMVEC/hMSC} \mathrm{CCC} \mathrm{cocul-}$ tures under vasculogenic culture conditions exhibited numerous capillaries, which were present in various stages of phenotypic maturation and differentiation. The typical linear capillaries were composed of elongated endothelial cells with thinned-out cytoplasm and oval to fusiform nuclei; the cells were characteristically in close apposition separated by narrow intercellular spaces, presumably a narrow slit-like luminal cavity (black arrows, Fig. 8A, B). The interdigitating cells revealed numerous plasmalemma vesicles, the caveolae, as well as electron-dense bodies (Fig. 8B, C). The overlapping cells also expressed the characteristic adherent and/or tight cell junctions (insets, Fig. 8C, D).

\section{Morphological and functional characterization of eCM and eCM/hMSC CCC cultures}

Immunocytochemical characterization (eCM CCCs). Immunolocalization of eCMs cultured on CCC scaffolds revealed that eCMs were able to adhere and undergo cellular coalescence, and were organized into tight sheets and/or clusters of predominantly elongated cells (Fig. 9A-H). 
A

\begin{tabular}{l} 
Collagen Cell Carrier - 3D Culture \\
\hline ahCMVECs Culture ahCMVECs and hMSCs Co-Culture
\end{tabular}

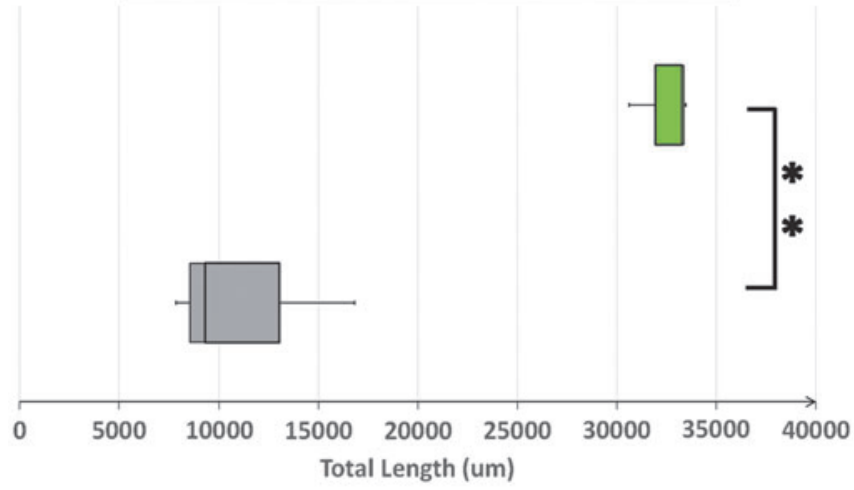

C

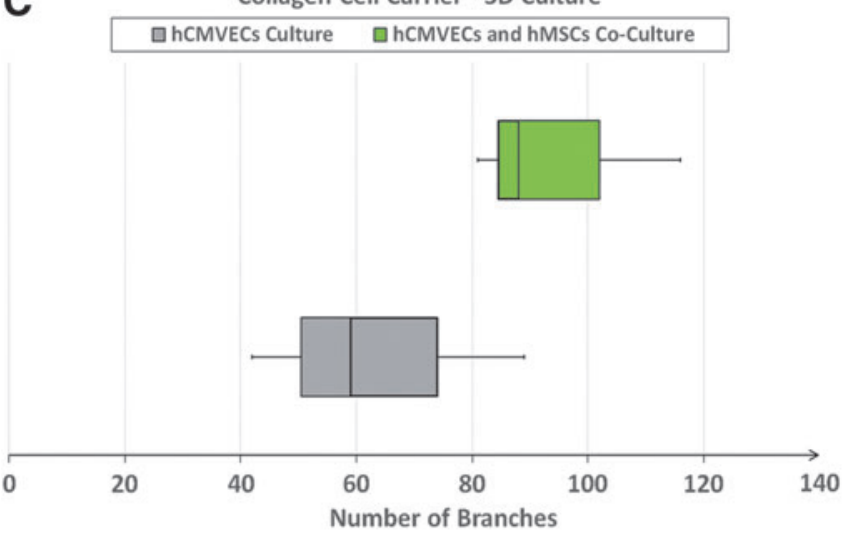

E

\begin{tabular}{l} 
Collagen Cell Carrier - 3D Culture \\
\hline ahCMVECs Culture $\square$ hCMVECs and hMSCs Co-Culture \\
\hline
\end{tabular}

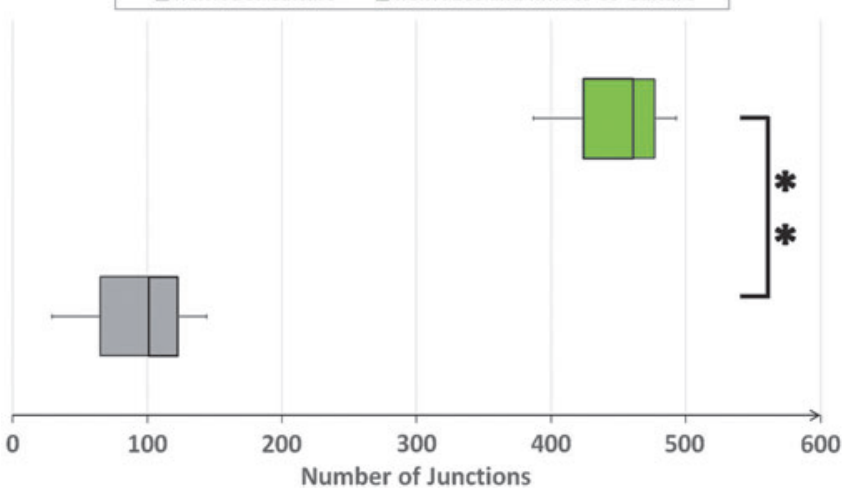

B

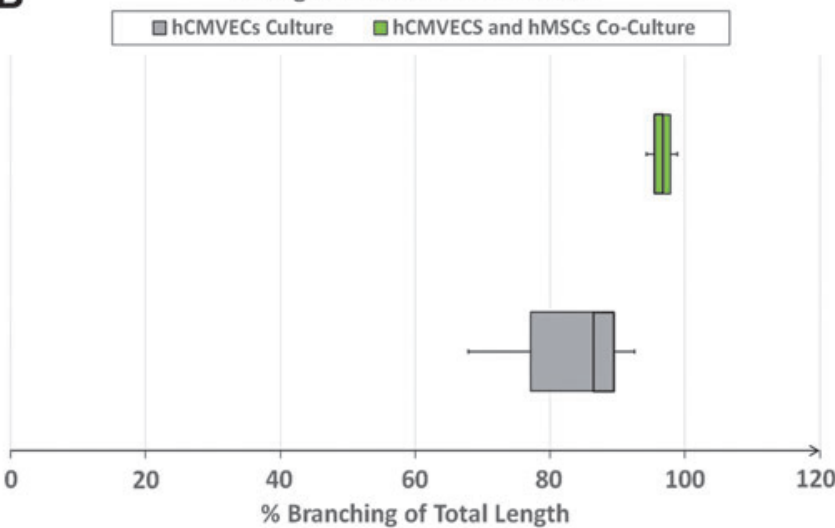

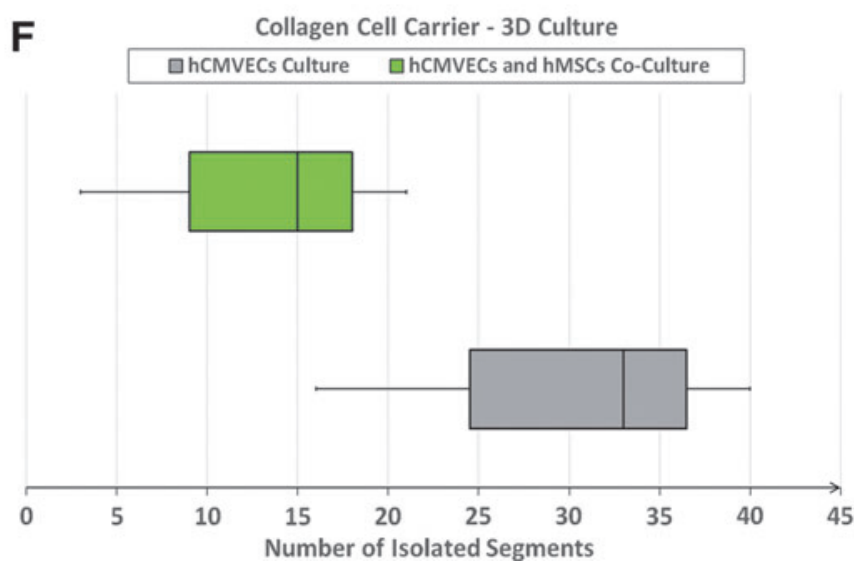

FIG. 5. Parameters characterizing the formation of endothelial cell microvascular networks in CCCs-hCMVEC culture versus hCMVEC/hMSC coculture. Comparison of various parameters characterizing the formation of endothelial cell microvascular networks, viz., total length (A), percentage $(\%)$ branching of total length $(\mathbf{B})$, number of branches $(\mathbf{C})$, number of meshes (D), number of junctions (E), and number of isolated segments (F), between day 14 vasculogenic hCMVEC CCC cultures and hCMVEC/hMSC CCC cocultures were analyzed using the ImageJ software (Angiogenesis Analyzer, an ImageJ plugin). The number of replicates, referring to number of microscopic images that were analyzed from three independent experiments $(n=10), * * p<0.001$.

These cells assumed mostly strap-like configuration with typical periodic cross-striations, were organized into multilayered functional syncytium, and also exhibited spontaneous beating and rhythmic contractions. The cells were typically positive for a set of cardiac myocyte-specific differentiation antigens, such as cardiac myosin heavy chain $(\alpha / \beta-M y h)$, desmin, cardiac troponin I (cTnI), Gata4, $F$-actin, brain natriuretic peptide (Bnp), gap junction pro- tein, connexin $45(C x 45)$, and $\alpha$-actinin (Fig. 9A-H and Supplementary Video S7), suggestive of functionally competent early-stage cardiac myocytes.

Cellular contractility (eCM/GFP-hMSCs CCCs). Phase contrast live-cell imaging using spinning disk confocal microscopy demonstrated that GFP-hMSCs were able to adhere to their neighboring beating myocytes, eCMs, within 
A Collagen Cell Carrier - 3D Co-Culture - hCMVECs and hMSCS 口Muscular Microvessels $\square$ Muscular Macrovessels

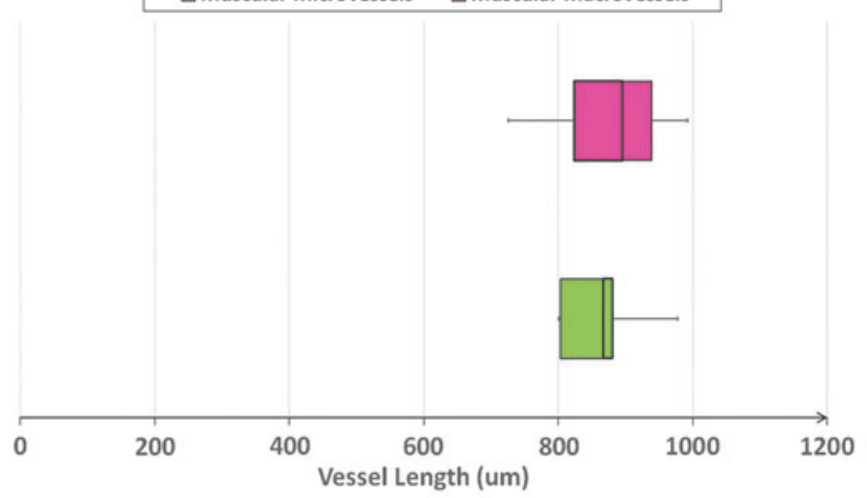

C Collagen Cell Carrier-3D-Co-Culture - hCMVECs and hMSCs (Muscular Microvessels)

口Area Covered by Endothelial Cells $\square$ Area Covered by Smooth Muscle Cells

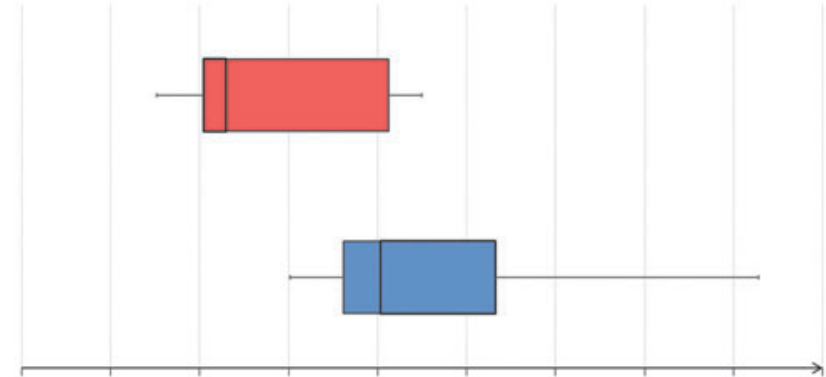

$0100002000030000400005000060000 \quad 7000080000 \quad 90000$ Area of Muscular Microvascular Cells $\left(\mathrm{um}^{2}\right.$ )
B Collagen Cell Carrier - 3D Co-Culture - hCMVECs and hMSCs 口Muscular Microvessels $\square$ Muscular Macrovessels

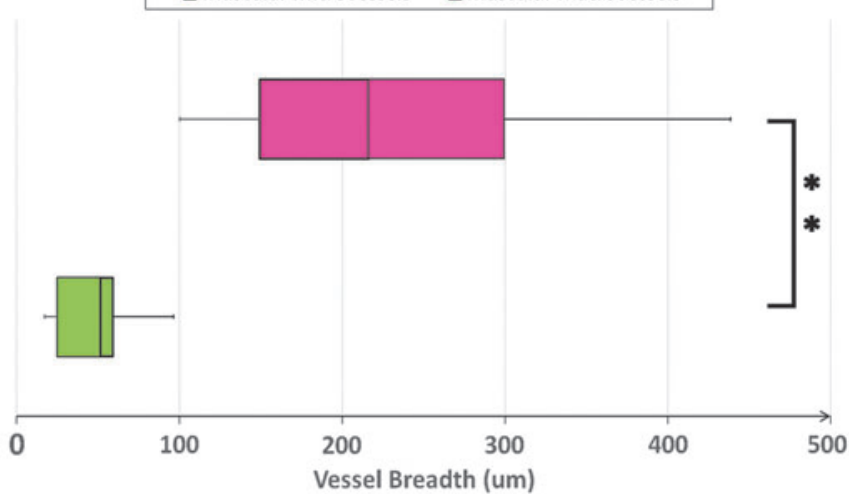

D Collagen Cell Carrier - 3D Co-Culture - hCMVECs and hMSCS (Muscular Macrovessels)

$\square$ Area Covered by Endothelial Cells $\square$ Area Covered by Smooth Muscle Cells

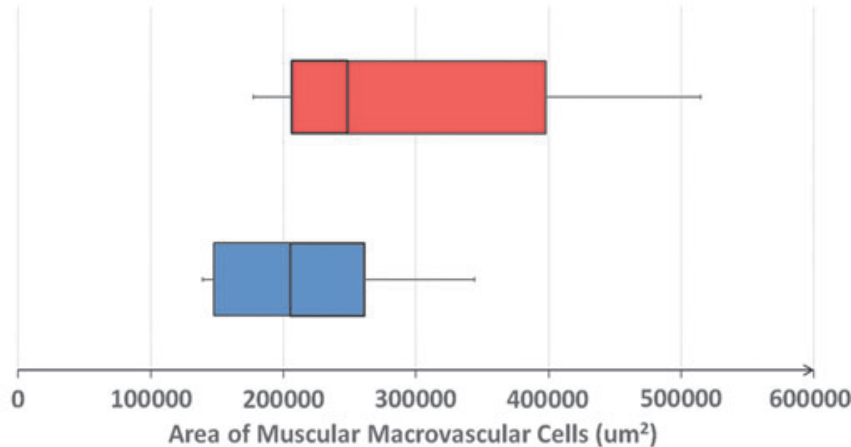

E

Collagen Cell Carrier - 3D Co-Culture - hCMVECs and hMSCs (Microvascular Networks)

口Area Covered by Endothelial Cells a Area Covered by Smooth Muscle Cells
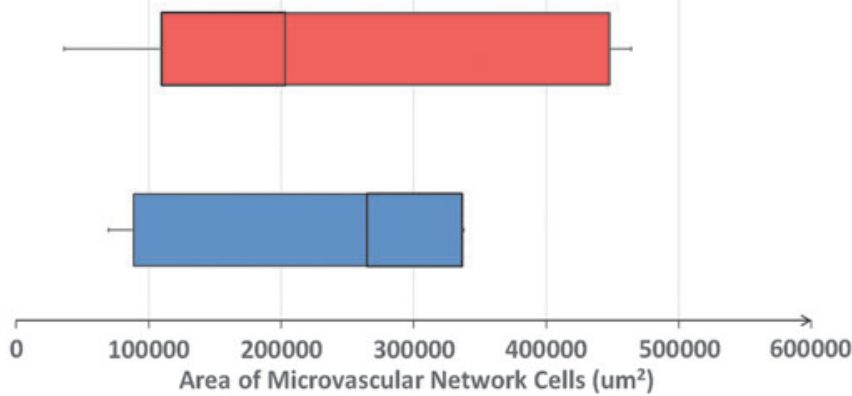

FIG. 6. Parameters characterizing the formation of large-caliber muscular vessels in CCCs-hCMVEC/hMSC coculture. Comparison of various parameters typifying the generation of both muscular microvessels $(>20-130 \mu \mathrm{m}$ wide) and muscular macrovessels $(>130 \mu \mathrm{m}$ wide), such as vessel length (A) and vessel breadth (B), in day 14 vasculogenic hCMVEC/hMSC CCC cocultures were analyzed using the ImageJ software. In addition, comparison of the areas covered by endothelial and smooth muscle cells was illustrated in $(\mathbf{C}-\mathbf{E})$ : muscular microvessels $(\mathbf{C})$, muscular macrovessels (D), as well as the microvascular networks $(\mathbf{E})$, that is, the small-caliber vessels $(<20 \mu \mathrm{m}$ wide). The number of replicates, referring to number of microscopic images that were analyzed from three independent experiments $(n=10), * * p<0.0001$.

a span of $24 \mathrm{~h}$ (Fig. 10A, B). The typical live-cell phase contrast image displayed that the mechanically coupled GFP-hMSCs were, in fact, able to undergo synchronized cellular contractions on par with that of eCMs (Fig. 10C, D). Assessment of GFP-hMSC active contraction/cellular movement relative to the active contraction exerted by its attached eCM established that, GFP-hMSCs were pri- marily in functional synchrony with that of eCMs (Fig. 10E, F). Thus, evidently, the cellular contractions were in unison, leading to a functional syncytium of the CCC construct (Supplementary Video S8). However, in this milieu, GFP-hMSCs that were not mechanically associated with eCMs or in standalone culture conditions (controls) revealed random noncyclical changes that were related in 

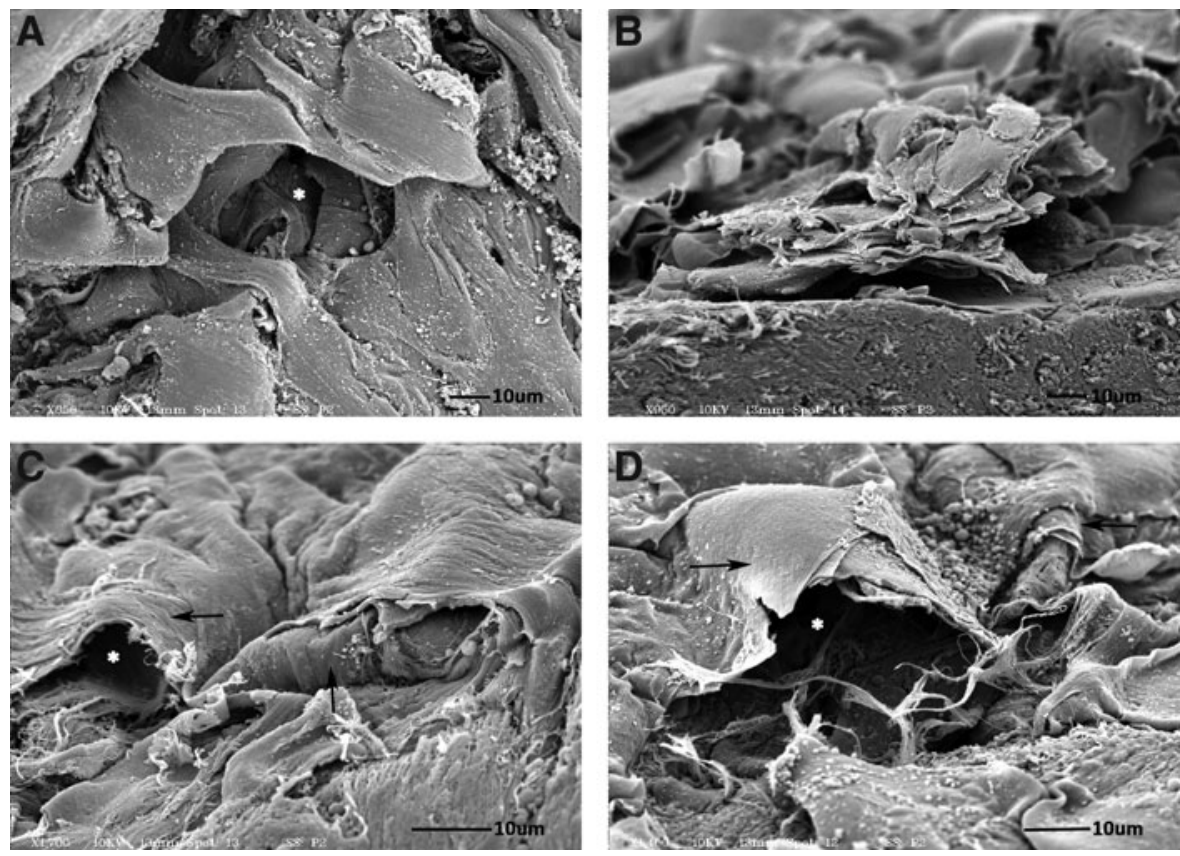

FIG. 7. SEM analysis of prevascularized CCCs-hCMVEC/hMSC coculture. SEM analysis of day $14 \mathrm{hCMVEC/hMSC}$ CCC cocultures in vasculogenic culture conditions exemplified a typical transverse capillary-like structure with its associated luminal cavity (white asterisk, A). The transverse-oriented capillaries were composed of flattened layer of differentiating cells. Furthermore, these differentiating cells demonstrated foci of multilayered cells, evoking cellular retraction and remodeling (B). In addition, existence of linear and convoluted tube-like structures with its attached mural type of cells (smooth muscle-like cells) was evident (black arrows, C, D). Some of these cylindrical structures displayed the presence of emerging patent lumens (white asterisks, C, D) or cavernous spaces (white asterisk, A). (A-D, scale bar 10 $10 \mathrm{~m})$. SEM, scanning electron microscopy.

FIG. 8. Ultrastructural characterization of prevascularized CCCs by TEM-hCMVEC/hMSC coculture. Ultrastructural characterization of day 14 hCMVEC/hMSC CCC vasculogenic cultures revealed capillaries that were present in various stages of maturation and differentiation. The elongated capillary structures were composed of endothelial cells with thinned-out cytoplasm; the cells were arranged on either side of a narrow slit-like luminal space (black arrows, A, B). Randomly oriented bundles of collagen fibers (col) of CCC were obvious on either side of these linear capillaries $(\mathbf{A}, \mathbf{B})$. Note the most characteristic feature of endothelial cells, that is, increase in the number of plasmalemma vesicles (B, C), and development of specialized cell junctions $(\mathbf{C}, \mathbf{D})$. The interdigitating endothelial cells expressed the characteristic adherent junction $(\mathbf{C}$, lower magnification; $\mathbf{D}$, higher magnification, and enlarged inset). (A, scale bar $10 \mu \mathrm{m} ; \mathbf{B}$, scale bar $2 \mu \mathrm{m} ; \mathbf{C}$, scale bar $500 \mathrm{~nm}$; D, scale bar $100 \mathrm{~nm}$ ). TEM, transmission electron microscopy.
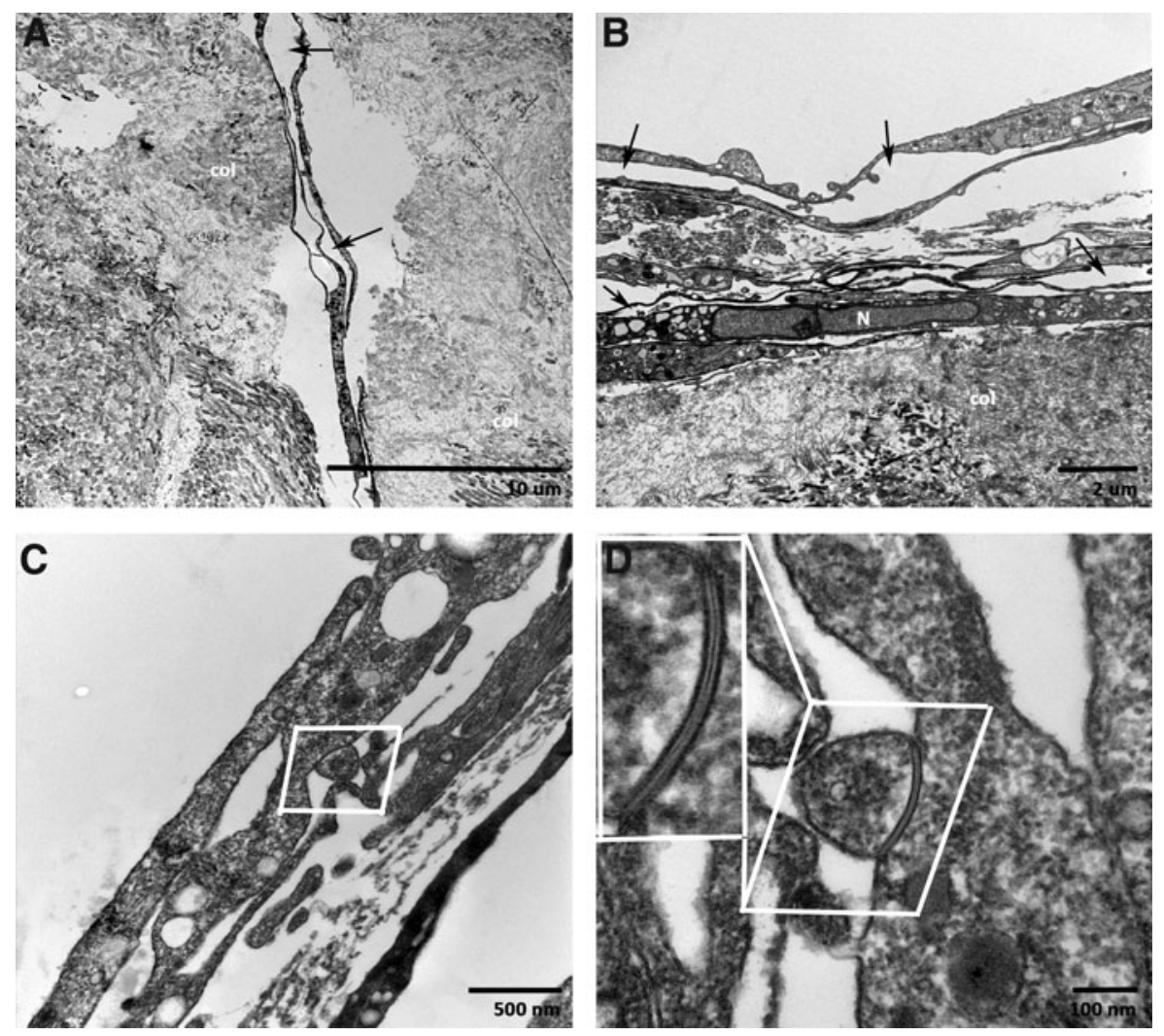

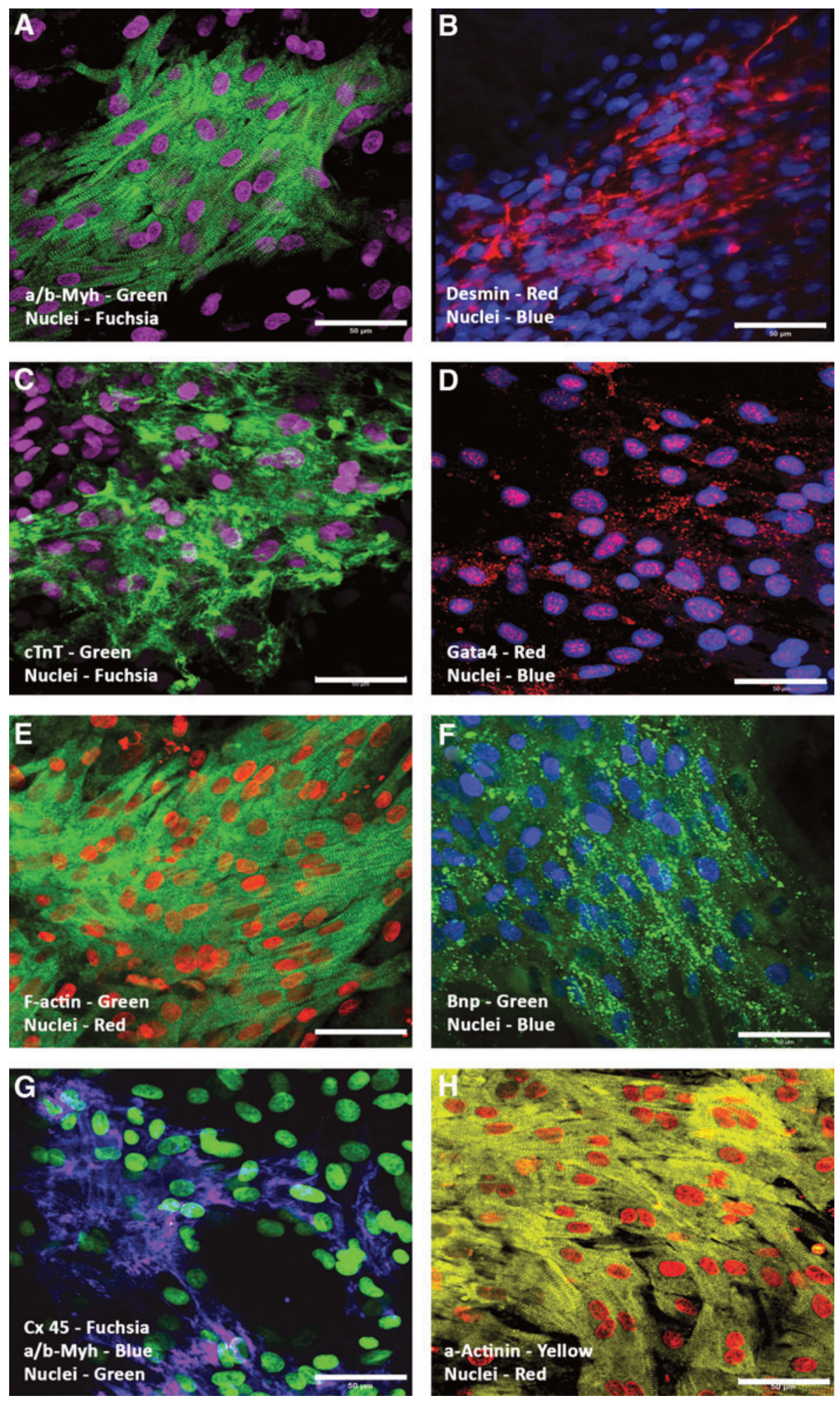

FIG. 9. Expression pattern of key cardiomyogenic markers in CCCs by immunofluorescence and confocal microscopy - eCM culture. Immunostaining of day $14 \mathrm{eCM}$ CCC cultures displayed the expression pattern of $\alpha / \beta-M y h$ (A), desmin (B), cTnT (C), Gata4 (D), F-actin (E), Bnp (F), Cx45 (G), and $\alpha$-actinin $(\mathbf{H})$. The CCCs showed coalition of myocytes; the myocytes were organized into sheets and clusters of multilayered functional syncytium. The cells appeared to be elongated and assumed strap-like configuration with periodic cross-striations; the nuclei of these cells were large and either round or oval in appearance, and were centrally situated. In some areas, the myocytes were overlapping with each other and were arranged in an orderly manner (AH). Cells were also counterstained for nuclei (blue, DAPI). Merged images (A-H). (A-H, scale bar $50 \mu \mathrm{m}) . \alpha / \beta-M y h$, cardiac myosin heavy chain; Bnp, brain natriuretic peptide; $C x 45$, connexin 45 ; eCMs, embryonic cardiac myocytes; Gata4, GATA binding protein 4. general with routine cellular migration and/or movements (data not shown).

Cellular calcium flux (eCM/GFP-hMSC CCCs). In general, excitation-contraction coupling of cardiac myocytes is known to be mediated by both the transsarcolemmal $\mathrm{Ca}^{2+}$ influx as well as intracellular sarcoplasmic reticulum $\mathrm{Ca}^{2+}$ release. To evaluate the existence of synchronous $\mathrm{Ca}^{2+}$ transients within the engineered CCC cardiac graft, we performed live-cell imaging studies with spinning disk confocal microscopy, using the free $\mathrm{Ca}^{2+}$ binding dyes, either Calcium Orange or Fluo-4.

To explore not only the eCM's intracellular calcium oscillations but also the codifferentiating hMSC's intracellular calcium flux, GFP-tagged hMSCs were cocultured with spontaneously and rhythmically beating eCMs. In this coculture conditions, the GFP-hMSCs that were mechanically coupled with eCMs displayed intracellular calcium spikes typified 

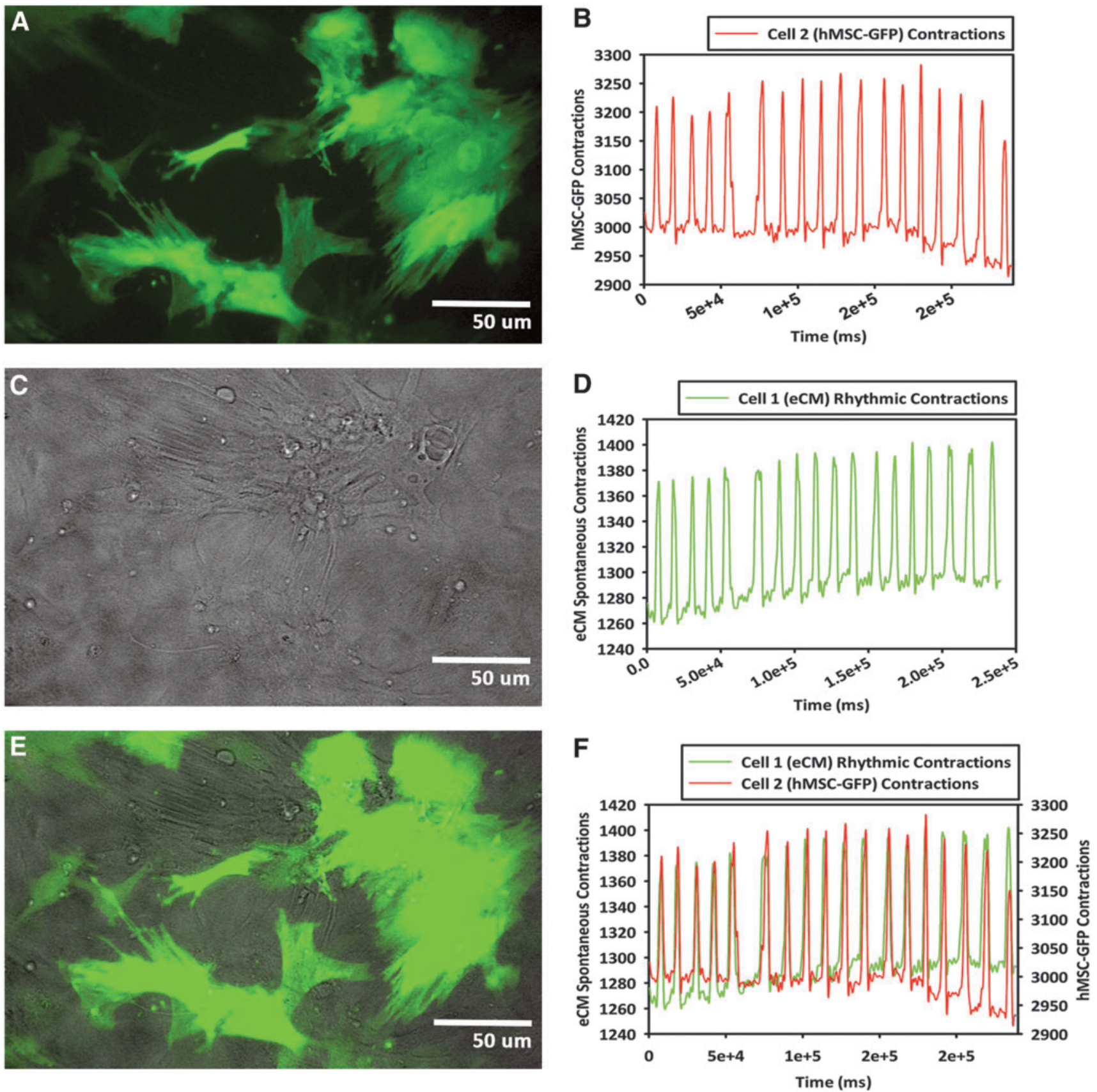

FIG. 10. Contractility assay of CCCs by live-cell imaging with spinning disk confocal microscopy-eCM/GFP-hMSC coculture. Phase contrast live-cell imaging using spinning disk confocal microscopy demonstrated that the fluorescently labeled hMSCs (GFP-hMSCs) were able to adhere to the juxtaposed beating eCMs. The mechanically coupled GFP-hMSCs were indeed able to contract in synchrony with its attached eCMs. The fluorescently labeled hMSCs that were attached with eCMs, shown in (A). The mechanically linked and transdifferentiated GFP-hMSC showed a typical pattern and frequency of cellular contraction and movement, shown in (B). The representative live-cell phase contrast image of GFP-hMSCs, which were mechanically coupled to eCMs, shown in $(\mathbf{C})$. The illustrative rhythmicity and pattern of contraction of an eCM, shown in (D). Superimposition of fluorescent and phase contrast images (A, C), highlighting the subtle spatial and temporal location of GFP-hMSCs and eCMs in the CCC coculture, displayed in (E). Assessment of GFP-hMSC active contraction/ cellular movement relative to the active contraction of its linked eCM established that GFP-hMSC was primarily in functional synchrony with its associated eCM. Thus, arguably, the cellular contractions were in unison, ultimately forming the required functional cardiac syncytium $(\mathbf{F})$. (A, C, E, scale bar $50 \mu \mathrm{m}$ ). GFP, green fluorescent protein. 

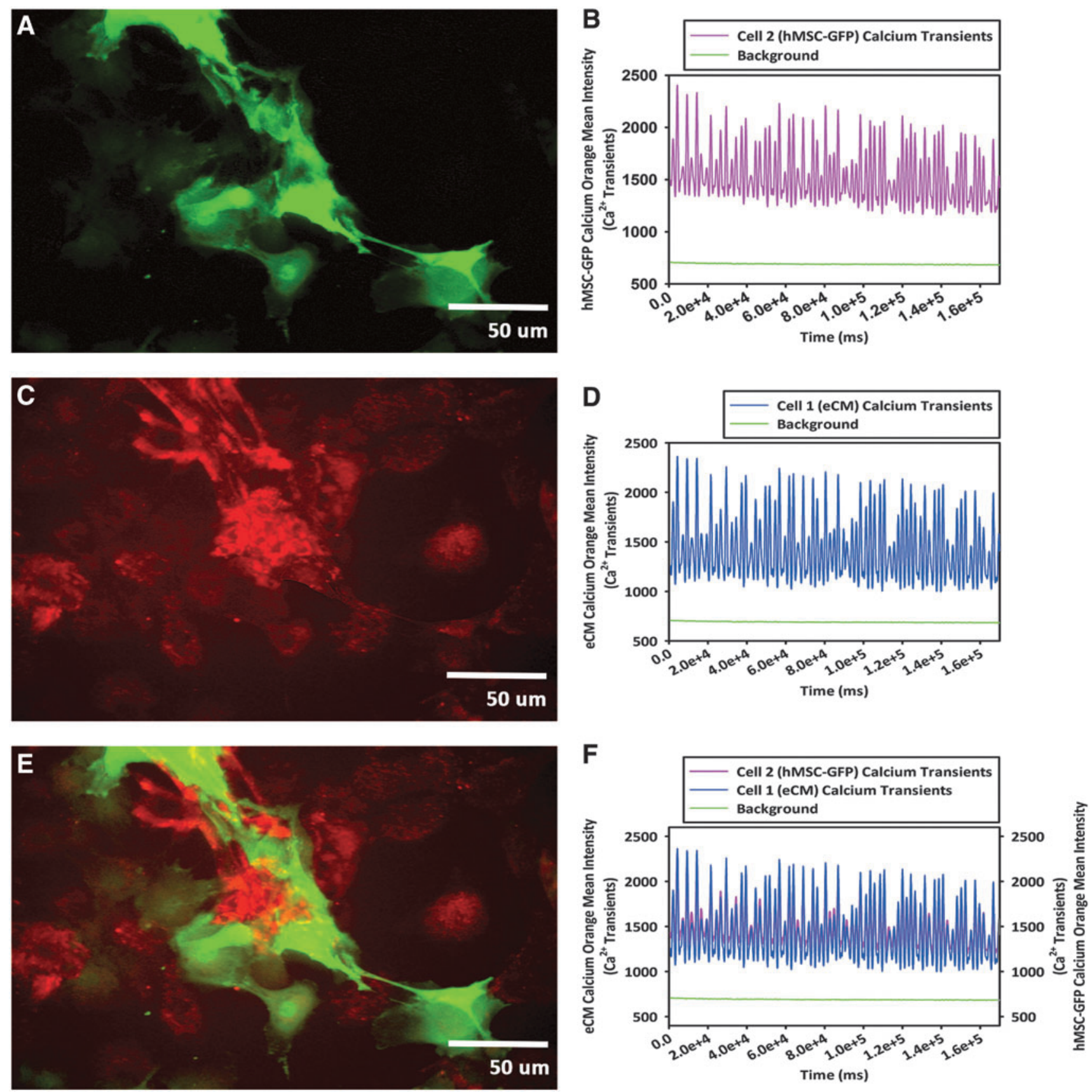

FIG. 11. Calcium flux assay of CCCs by live-cell imaging with spinning disk confocal microscopy-eCM/GFP-hMSC coculture. Live-cell imaging of intracellular calcium flux using spinning disk confocal microscopy revealed that the fluorescently labeled hMSCs (GFP-hMSCs) were, in fact, able to tether to the surrounding spontaneously contracting eCMs, and imaged in the green channel, depicted in (A). The GFP-hMSC displayed a particular pattern and frequency of calcium oscillations, shown in (B). The amplitude of the GFP-hMSC's calcium flux was relatively smaller than the coupled myocyte, shown in (D, F), indicating that the GFP-hMSCs were able to develop their contractile apparatus, which were almost on par with that of eCMs. The calcium indicator, Calcium Orange, labeled eCMs and GFP-hMSCs, imaged in the red channel, shown in (C). The calcium flux of a spontaneously and electrically pacing eCM, shown in (D). Overlap images of GFPhMSCs and eCMs, both labeled with the calcium indicator, Calcium Orange, and imaged in the red channel, illustrated in (E). Examination of the calcium oscillations of GFP-hMSC in correspondence with that of its mechanically coupled and spontaneously beating eCM demonstrated that the pattern and frequency of contraction of both these interacting cells were essentially the same (F). Hence, suggesting that GFP-hMSCs were not only mechanically coupled but also electrically coupled to the juxtaposed and examined eCMs, however, arguably, with relatively smaller spikes, as illustrated in (F). (A, $\mathbf{C}, \mathbf{E}$, scale bar $50 \mu \mathrm{m})$. 

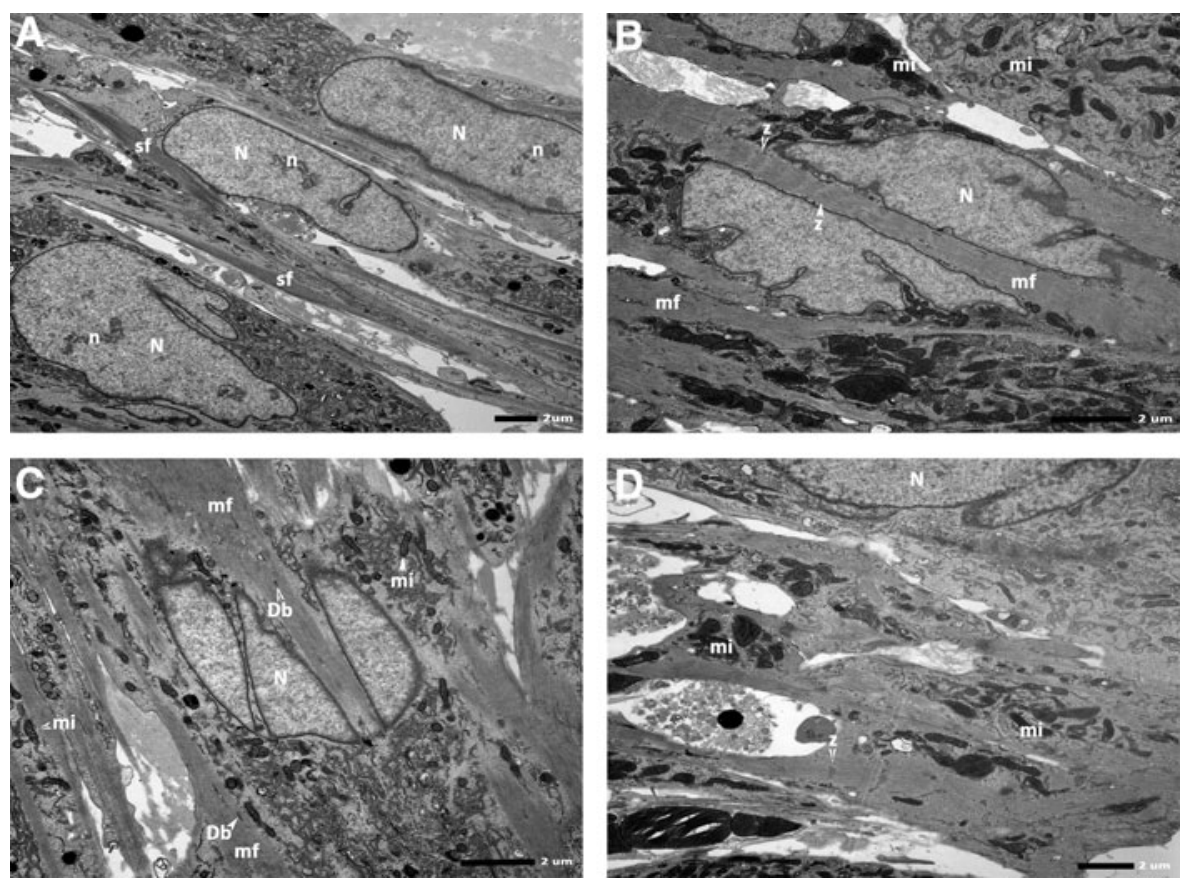

FIG. 12. Ultrastructural characterization of avascular cardiac graft by TEM analysis-hMSC culture, eCM culture, and $\mathrm{eCM} / \mathrm{hMSC}$ coculture. TEM analysis of hMSC CCC cultures displayed the ultrastructural features of a typical somatic stem cell. The undifferentiated cytoplasm of hMSCs expressed widespread endoplasmic reticulum and several mitochondria, as well as bundles of actin stress fibers (sf) principally organized toward the marginal cytoplasm. The nuclei were euchromatic (N) with single to multiple active nucleoli (n). The nuclei assumed stretched-out configuration and were centrally positioned in the cytoplasm (A). Likewise, analysis of eCM CCC cultures under similar culture conditions demonstrated the ultrastructural characteristics of early stages of developing myocytes. These cells showed evolving myofibrillar organization, including disorganized myofibrils (mf), perinuclear assembly of early sarcomeric units, Z-discs (Z), as well as abundant pleomorphic mitochondria (mi) interspersed among the myofibrils along with glycogen granules (B). On the contrary, the $\mathrm{eCM} / \mathrm{hMSC}$ CCC cocultures revealed interdigitation and/or fusion of juxtaposed hMSCs and eCMs, and lack of detectable cellular demarcation was evident $(\mathbf{C})$. The myofibrillar organization was disorganized and/or remodeled in places, and were reoriented in a particular direction. The cytoplasm of these coupled cells expressed a number of stretched-out myofibrils, along with randomly arranged tubules of sarcoplasmic reticulum, scattered pleomorphic mitochondria (mi), as well as widespread glycogen particles (D). The codifferentiating cells depicted typical imperfections of the myofibrillar organization, exemplified by discontinuous and/or widened Z-discs, electron dense bodies (Db, Z-bodies), indistinct $\mathrm{H}$ zones, and free-floating myofibrils (C, D). Besides, some differentiating myocytes exhibited myofibrils with regular Z disks (Z) and distinct $\mathrm{A}$ and I bands (D). Consequently, these characteristics were reminiscent of partial dedifferentiation followed by redifferentiation of these mechanically coupled and physically interacting cells in this milieu. (A-D, scale bar $2 \mu \mathrm{m})$.

FIG. 13. mRNA expression profiles of various key vasculogenic and cardiomyogenic markers by RT-qPCR-hCMVEC culture, hCMVEC/hMSC coculture, eCM culture, and eCM/hMSC coculture. mRNA expression profiles of various key vasculogenic differentiation markers, such as PECAM1, KDR, TIE1, TEK, and VWF expression ( $x$-axis), as a function of time ( $y$-axis). hCMVECs cultured onto CCCs under vasculogenic culture conditions (A). hCMVECs/hMSCs cocultured onto CCCs under vasculogenic culture conditions (B). Likewise, mRNA expression profiles of various key cardiomyogenic differentiation markers, such as Myh6, Myh7, Actc1, Tnni3, Gata4, Nppa, Nppb, and Gjal expression ( $x$-axis), as a function of time ( $y$-axis). eCMs cultured onto CCCs under myogenic culture conditions (C, $\mathbf{E})$. eCMs/hMSCs cocultured onto CCCs under myogenic culture conditions $(\mathbf{D}, \mathbf{F})$. The target gene expression was normalized by three nonregulated reference gene expressions, such as Gapdh/GAPDH, Actb/ $\beta$-actin, and either G6pd/G6PD or RplpO/RPLPO. The calibrator controls included hCMVECs day 0 and eCMs day 0 samples for vasculogenic and cardiomyogenic cultures, respectively. The expression ratio ( $x$-axis) was calculated using the Relative Expression Software Tool-384 (REST-384 ${ }^{\odot}$, version 2 ). The values were means \pm standard errors for three independent cultures $(n=3), * p<0.05 ; * * p<0.001$. Actb/ $\beta$-actin, Actin, beta; Actc1, actin, alpha, cardiac muscle 1; G6pd/G6PD, glucose-6-phosphate dehydrogenase; Gapdh/GAPDH, glyceraldehyde3-phosphate dehydrogenase; Gjal, gap junction protein, alpha 1; KDR, kinase insert domain receptor, a type III receptor tyrosine kinase; mRNA, messenger RNA; Myh6, myosin heavy chain 6; Myh7, myosin heavy chain 7; Nppa, natriuretic peptide A; $N p p b$, natriuretic peptide B; Rplp0/RPLPO, ribosomal protein lateral stalk subunit P0; RT-qPCR, reverse transcription-quantitative real-time polymerase chain reaction; TEK, TEK tyrosine kinase, endothelial; TIE1, tyrosine kinase with immunoglobulin-like and EGF-like domains 1; Tnni3, troponin I3, cardiac type. 
by a cyclic increase of the cytosolic $\mathrm{Ca}^{2+}$ concentration and a subsequent removal of $\mathrm{Ca}^{2+}$ as illustrated (Fig. 11A, B). On imaging over a period of $5 \mathrm{~min}$ or longer, GFP-hMSC that was mechanically attached to a spontaneously and electrically pacing neighboring eCM (Fig. 11C, D) exhibited intracellular calcium oscillations/spikes, which resembled the intracellular calcium spikes of the coupled eCM (Fig. 11E, F). However, in control CCCs, that is, GFPhMSCs, which were not under coculture conditions exhibited insignificant intracellular calcium flux (bottom green trace, Fig. 11B, D, F).
Ultrastructural characterization of avascularized cardiac CCCs (hMSC, eCM, and eCM/hMSC cultures)

TEM analysis of hMSC CCC cultures displayed the ultrastructural features of a typical postnatal stem cell. In these cultures, hMSCs were elongated and their undifferentiated cytoplasm revealed scattered numerous mitochondria and extensive endoplasmic reticulum, as well as centrally positioned stretched-out nuclei, depicting opened-out chromatin with multiple nucleoli. All these characteristics were consistent with a primitive and naive mesenchymal type of cell.

\section{Markers of Vascular Differentiation - mRNA Analysis}

A

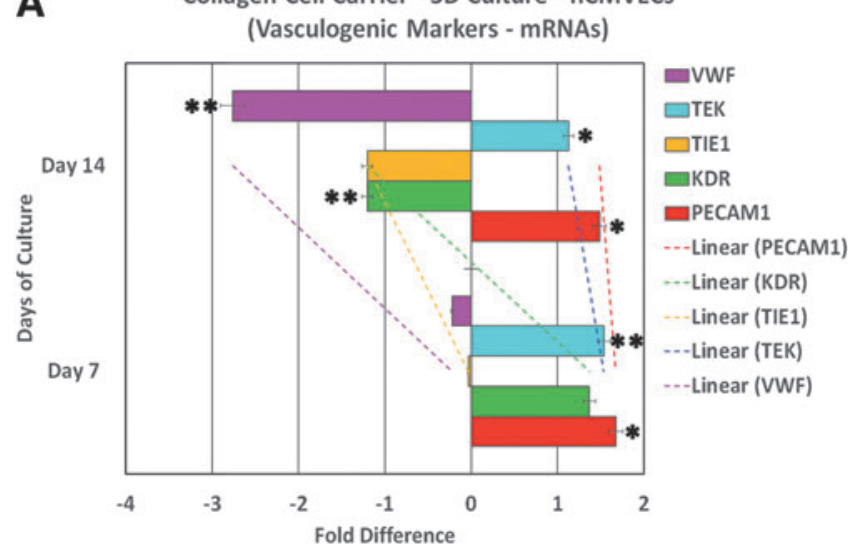

B Collagen Gel Carrier - 3D Co-Culture - hCMVECs and hMSCs (Vasculogenic Markers - mRNAs)

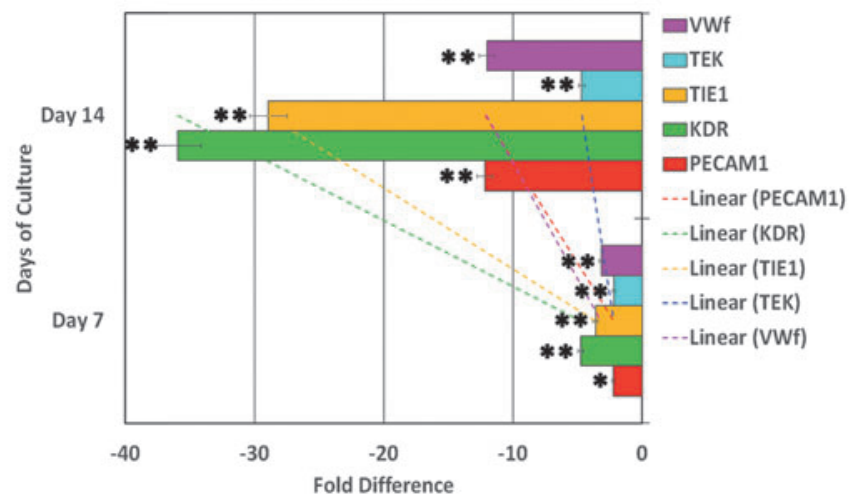

Markers of Cardiomyocyte Differentiation - mRNA Analysis

Collagen Cell Carrier - 3D Culture - eCMs
(Cardiomyogenic Markers - Filamental mRNAs)

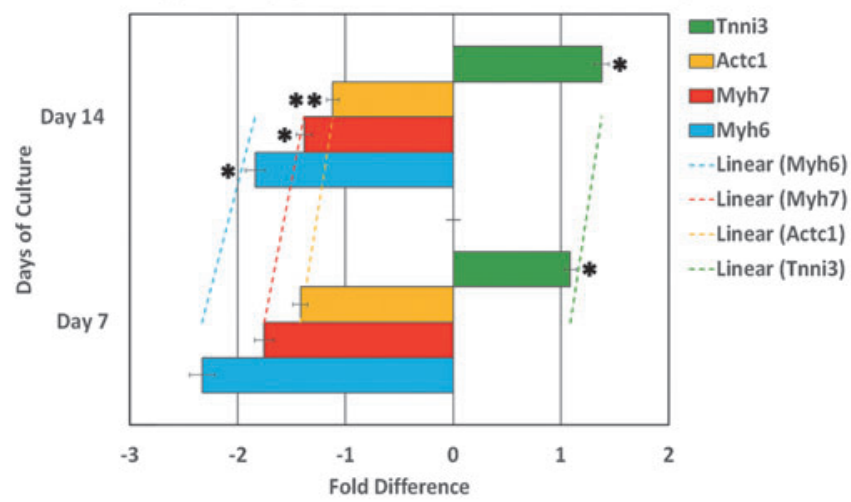

E

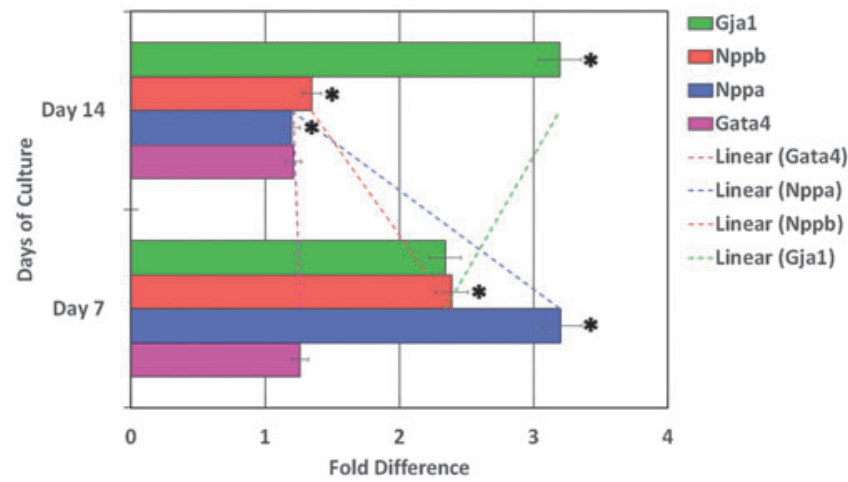

D Collagen Cell Carrier - 3D Co-Culture - eCMs and hMSCs (Cardiomyogenic Markers - Filamental mRNAs)

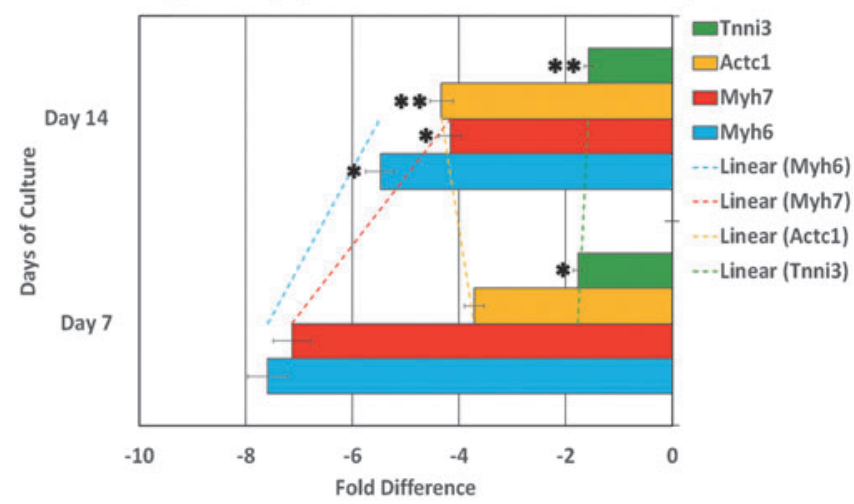

F Collagen Cell Carrier - 3D Co-Culture - eCMs and hMSCs (Cardiomyogenic Markers - Non-Filamental mRNAs)

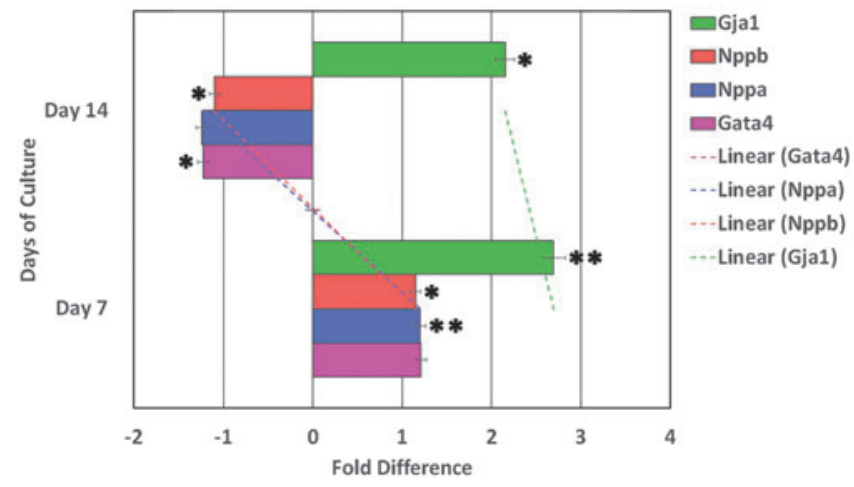


However, in addition, these cells expressed bundles of actin stress fibers primarily organized at subplasmalemmal region (Fig. 12A). Equally, analysis of eCM-only CCC cultures depicted the ultrastructural characteristics of early stages of developing myocytes. The cells revealed emerging myofibrillar organization, including certain degree of randomly positioned myofibrils, mostly perinuclear assembly of early sarcomeric units, Z-discs, as well as pleomorphic mitochondria and glycogen particles interspersed among the myofibrillar organization (Fig. 12B).

In contrast, the eCMs/hMSCs containing CCCs showed tight adherence of juxtaposed hMSCs and eCMs, and in some areas, lack of detectable cellular boundaries between them (Fig. 12C, D). The myofibrillar architecture was disorganized and/or remodeled in places, and were oriented in a particular direction. The cytoplasm of these mechanically coupled cells expressed a number of stretched-out myofibrils, along with numerous randomly positioned pleomorphic mitochondria and widespread glycogen granules.

The codifferentiating cells revealed characteristics of cell-cell interaction and mechanotransduction features, such as typical imperfections of the myofibrillar organization due to cyclic stretching and remodeling, typified by discontinuous and/or widened Z-discs, evolving electron dense bodies ( $\mathrm{Z}$ bodies), indistinct $\mathrm{H}$ zones, and free floating myofibrils, a sign of partial dedifferentiation followed by redifferentiation of these mechanically coupled and physically interacting cells in this milieu. Apart from this, certain codifferentiating cells demonstrated myofibrils with regular Z-discs and distinct $\mathrm{A}$ and I bands (Fig. 12C, D), a sign of progressive maturation and differentiation toward neonatal and/or juvenile type of myocytes.

\section{Differential gene expression profile of various CCC cultures-messenger RNA analysis of lineage-specific differentiation markers}

First, to compare the differential gene expression profile between hCMVEC/hMSC CCC cocultures versus hCMVEC CCC cultures, RT-qPCR of key vasculogenic differentiation markers were performed using day 7 and 14 messenger RNA (mRNA) samples (Fig. 13A, B).

In hCMVEC CCC vasculogenic cultures, expression of $P E C A M 1, K D R$, and TEK remained upregulated during day 7; subsequently, all these three genes' transcripts showed a progressive downregulation on day 14. The other two genes, viz., TIE1 and VWF showed slight downregulation on day 7 , and sustained their downregulation until day 14 (Fig. 13A). In comparison, hCMVEC/hMSC CCC vasculogenic cocultures, demonstrated slight downregulation of all the key vasculogenic genes, PECAM1, KDR, TIE1, TEK, and VWF during day 7 , and remained downregulated till day 14 , significantly (Fig. 13B). Most importantly, out of all the assessed vasculogenic genes, the $K D R$ and $T I E 1$ showed remarkable level of downregulation around day 14 .

Next, to compare the differential gene expression profile between eCM/hMSC CCC cocultures versus eCM CCC cultures, RT-qPCR analyses of key cardiomyogenic differentiation markers were performed on day 7 and 14 mRNA samples (Fig. 13C-F).

Myogenic eCM CCC cultures demonstrated an initial downregulation of cardiac-specific structural and contractile genes, viz., Myh6, Myh7, and Actcl on day 7, and subsequently, their expression was progressively upregulated during day 14. The other remaining contractile filament-associated gene, Tnni3, showed an initial upregulation on day 7 and its expression remained upregulated until day 14 (Fig. 13C). In addition, the same eCM CCC cultures, on examination, showed that the nonfilamental cardiac-specific genes, such as Gata4, Nppa, and Nppb showed an initial upregulation on day 7 , and their expression seemed to downregulate around day 14. On the other hand, the gap junction gene, Gjal, revealed an initial upregulation on day 7 , with noticeable kinetics of sustained upregulation of its transcripts over the observed consecutive time point, that is, around day 14 (Fig. 13E).

Compared to eCM CCC cultures, in eCM/hMSC CCC cocultures, Myh6, Myh7, and Tnni3, that is, the cardiacspecific structural and contractile genes, demonstrated an initial downregulation on day 7 , and their expression reminded progressively upregulated until day 14 . While, the other remaining contractile element, Actc1, showed an initial downregulation on day 7 followed by continued, but slight downregulation on day 14 (Fig. 13D). Furthermore, in eCM/hMSC CCC cocultures, the cardiac-specific nonfilamental genes, such as Gata4, Nppa, Nppb, and Gjal, displayed a noticeable initial upregulation of their mRNA transcripts around day 7, and later, their expression levels became progressively downregulated on day 14 (Fig. 13F).

Finally, the observed patterns of differential gene expressions, in these disparate vasculogenic and myogenic culture conditions, were not only indicative of cell-cell interactions but also suggestive of cell-matrix interactions, which ultimately led to the modulation of vasculogenic and cardiomyogenic gene expressions, and could be attributed to the here and now physicochemical environmental cues, and consequently, the differentiation potentials of these cells on the CCC scaffold.

\section{Characterization of vascularized cardiac graft using lineage-specific markers}

Immunolocalization of tri-cell (hCMVEC/eCM/hMSC) CCC cocultures using the same set of lineage-specific markers, as indicated above, was performed. Comparable to what was seen within eCM CCC culture (Fig. 9), the codifferentiating cells were positive for a battery of key cardiac myocyte differentiation markers, including $C \times 43$, desmin, cTnT, $\alpha / \beta$-Myh, $N$-Cadherin, cTnI, and F-actin (Fig. 14A-F). These cells were assembled into a functional syncytium by means of cellular coalescence and alignment. The cells were elongated and stretched out, and organized into interweaving latticework of myocytes, revealing the $N$ Cadherin-positive intercalated discs and desmin-positive Zbands. Besides, these cells were positive for Connexin 43 and 45 , the gap junction proteins, indicative of various stages of phenotypic maturation and differentiation of myocytes. The characteristic staining pattern that captured the myocytespecific proteins within the vascularized cardiac grafts are shown in Figure 14A-F.

On further examination, amidst the interdigitating layers of myocytes, comparable to what was observed within hCMVEC/hMSC cocultures (Figs. 2 and 3), the codifferentiating cells were positive for a set of endothelial and smooth muscle markers, for instance, VWF and LECTIN (Fig. 14G, H). By differential adhesion-driven cell sorting 

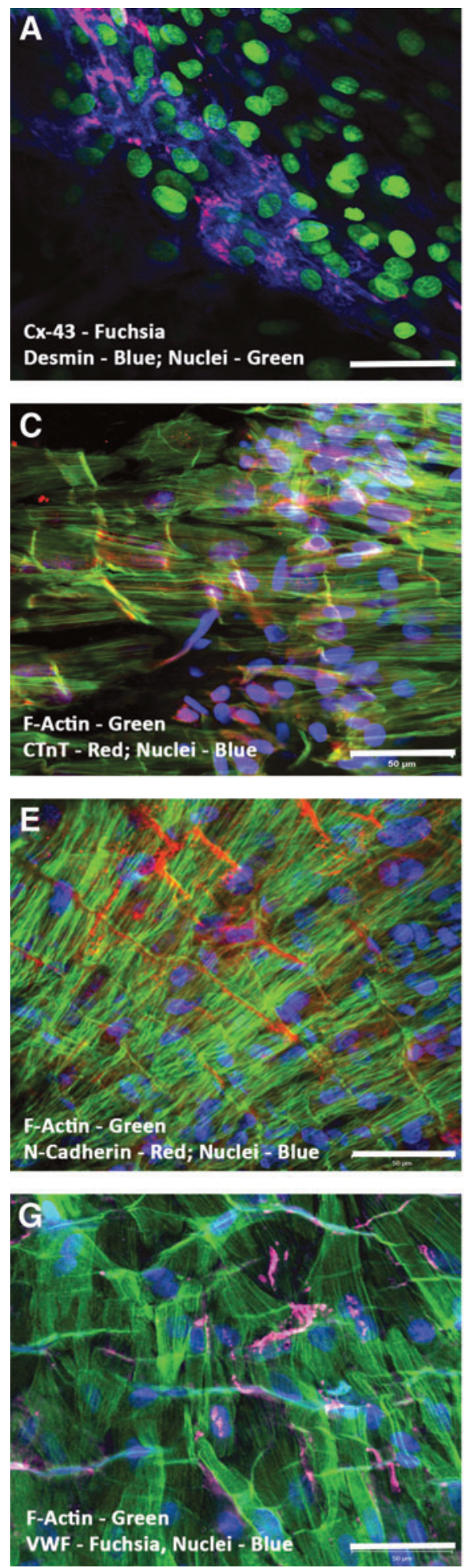
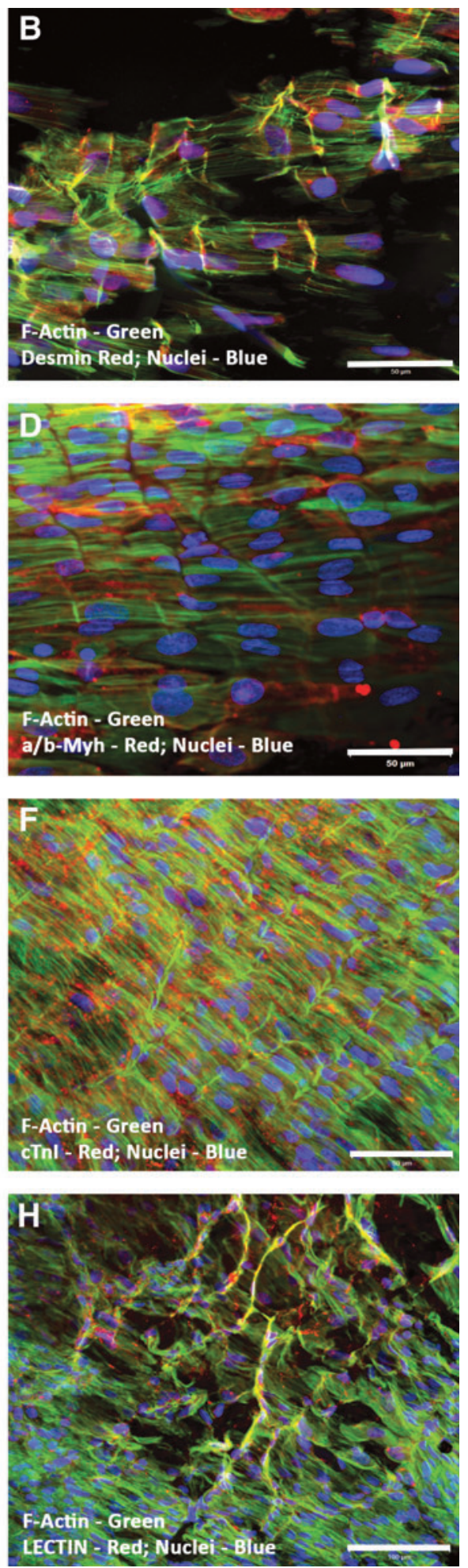

FIG. 14. Characterization of vascularized cardiac graft by immunofluorescence and confocal microscopy-hCMVEC/eCM/ hMSC tri-cell coculture. Immunostaining and confocal microscopic analyses of tri-cell (hCMVEC/eCM/hMSC) CCC cocultures consistently displayed the expression of several key cardiomyogenic and vasculogenic differentiation markers. The differentiating cells were positive for $C x 43$ (A), desmin (B), $c \operatorname{TnT}$ (C), $\alpha / \beta$-Myh (D), $N$-Cadherin (E), $c \operatorname{TnI}(\mathbf{F})$, and $F$-actin $(\mathbf{B}-\mathbf{H})$. The cells were assembled into a functional syncytium, consisting of mostly strap-like myocytes with cross-striations and orderly registry of sarcomeres. On examination, amidst the interdigitating and latticework of myocytes were seen the VWF- $(\mathbf{G})$ and LECTIN- $(\mathbf{H})$ positive vascular cells arranged predominantly in linear and/or plexuses of dense vascular networks. The nuclei of these vascular cells were smaller and either elongated or fusiform in shape, and could be readily delineated from the nuclei of the myocytes, which were large, either oval or round in nature, and were centrally located. Cells were also counterstained for nuclei (blue, DAPI); and fibrillary actin with phalloidin (green, Alexa 488 phalloidin). Merged images (A-H). (A-E, scale bar $50 \mu \mathrm{m} ; \mathbf{F}-$ H, scale bar $100 \mu \mathrm{m})$. $c T n I$, cardiac troponin I; Cx43, connexin 43. and relative positioning, these vascular cells were selfassembled into an extensive network of linear and/or branching capillaries. The nuclei of these vascular cells were smaller and assumed either stretched-out or fusiform configuration, and could be readily delineated from the nuclei of the myocytes, which were large and either oval or round in nature, and were centrally situated. The representative staining pattern that captured the endothelium-related proteins within the vascularized cardiac grafts is illustrated in Figure 14G and $\mathrm{H}$.

\section{Chronotropic and ionotropic responses}

of the engineered vascularized cardiac graft

Evaluation of vascularized cardiac grafts composed of all the three categories of cells (viz., hCMVECs/eCMs/ hMSCs), in response to application of cardioselective pharmacological agent-revealed characteristic patterns of calcium oscillations, hence the contractile responses (Supplementary Videos S9 and S10). Application of a nonselective $\beta$ - 
adrenoceptor agonist, IPN, resulted in an increase in frequency and amplitude of calcium oscillations, demonstrating an increase in the myocyte beating rate and its increased contractile force, suggestive of typical elevation in chronotropic and ionotropic responses. (Fig. 15A, B). However, application of a calcium channel blocker, DTZ, to a similar sample, the beating cardiac patch, resulted in an opposite effect, progressively attenuated the frequency and amplitude of calcium oscillations, demonstrating a weakened myocyte beating rate and its decreased contractile force, indicative of typical decreased chronotropic and ionotropic effects (Fig. 15G).

In contrast, the application of a sympathomimetic amine, $\beta_{2}$-agonist, CLEN, led to a raise in the frequency of calcium flux, consequently, an increase in the myocyte beating, that is, an elevated chronotropic effect (Fig. 15D, E). Sequential application of drugs, such as initial application of IPN to a beating CCC cardiac construct resulted in elevated chronotropic and ionotropic responses (red arrow). The successive application of DTZ to the same sample manifested in sustained negative chronotropic and ionotropic responses (Fig. 15C). Similarly, the initial application of CLEN to a spontaneously beating cardiac construct displayed augmented ionotropic and chronotropic responses, while a subsequent application of diltiazem showed sustained negative chronotropic and ionotropic effects (Fig. 15F).

Quantitative analysis of the influence of $\beta$-adrenoceptor activation or L-type calcium channel inhibition of vascularized cardiac grafts, validated that the amplitude of intracellular calcium oscillations due to the effect of $\beta$ adrenoceptor activation by IPN exposure $\left(\Delta \mathrm{Ca}^{2+}=2067 \pm\right.$ 64) was significantly greater $(p=0.001)$ than that seen before IPN exposure $\left(\Delta \mathrm{Ca}^{2+}=1639.2 \pm 61\right)$ (Fig. 16A). However, no statistically significant difference $(p=0.802)$ was observed with $\left(\Delta \mathrm{Ca}^{2+}=1680.67 \pm 170\right)$ or without $\left(\Delta \mathrm{Ca}^{2+}=\right.$ $1641.25 \pm 39)$ the addition of CLEN on the spontaneously beating cardiac grafts (Fig. 16B). Furthermore, the addition of L-type calcium channel blocker, DTZ, resulted in the diminution of the amplitude of intracellular calcium oscillation $\left(\Delta \mathrm{Ca}^{2+}=1454 \pm 43\right)$, and was significantly lower $(p=0.011)$ than that seen before DTZ addition $\left(\Delta \mathrm{Ca}^{2+}=\right.$ $1640 \pm 35$ ) (Fig. 16C).

These results clearly demonstrated that the brisk response of cardiac grafts could be elicited by the application of various cardioactive drugs, and consequently, the functional competence of the tissue construct.

\section{Discussion}

In this study, we report a reproducible and exemplary in vitro $3 \mathrm{D}$ model of mammalian cardiomyogenesis that can be utilized to dissect various molecular mechanisms that are underpinning the orderly cellular differentiation and tissue morphogenesis. The generated 3D cardiac tissue recapitulates several aspects of in vivo neo-vasculogenesis and neocardiomyogenesis. We have demonstrated unequivocally that cardiac endothelial-myocardial interaction or signaling is a prerequisite for successful development of tissueengineered cardiac graft. Also, we have shown the integrative aspect of hMSCs, and discernable excitation and contraction coupling between myocytes and stem cells, and ultimately, its fate determination. Consequently, we have revealed the temporal and spatial regulation of codifferentiating hCMVECs (microvascular endothelial cells) and eCMs (myocytes) in our natural biomimetic scaffold, CCC, in the presence of somatic stem cells, hMSCs.

The mosaic of myocardial tissue recreated by means of modular and bottom-up tissue engineering approaches reiterated not only several crucial aspects of in vivo neovasculogenesis and neo-cardiomyogenesis but also enabled us to critically evaluate the controversial aspects of the role of hMSCs on the induction/differentiation of eCMs, and most significantly, whether hMSCs had multilineage differentiation potential in this milieu, and could transdifferentiate into myocytes, endothelial cells, and smooth muscle cells, undoubtedly.

Development of vasculature, that is, assembling an in situ de novo primitive vascular network, is one of the earliest events that occurs during embryogenesis, and preceding organ formation. Organogenesis requires a multifaceted set of paracrine signals between the vasculature and the developing nonvascular tissues to support cellular differentiation and growth of an organ. ${ }^{38}$ However, it has recently been recognized that endothelial cells play a vital role not only in regulating and maintaining tissue-specific cells but also controlling organ growth and ultimately, its size. ${ }^{1,38}$ This implies that angiogenesis and vascular invasion are a prerequisite to the process of tissue morphogenesis both in development and repair.

The human heart is a highly organized pluricellular, multifunctional organ, consisting of diverse and equipotent cell types, for example, cardiac myocytes, cardiac fibroblasts, endothelial cells, smooth muscle cells, as well as

FIG. 15. Pharmacological response of vascularized cardiac graft by live-cell imaging with spinning disk confocal microscopy. Calcium oscillations of the spontaneously beating CCC cardiac grafts were evaluated following the application of different cardioactive pharmacological agents $(0.1-1 \mu \mathrm{M})$. Characteristic calcium oscillations recorded in the case of spontaneously and rhythmically contracting cells, that is, before the application of a nonselective $\beta$-adrenoceptor agonist, isoprenaline (A). Calcium flux in response to isoprenaline application expressed typical elevation of chronotropic and ionotropic responses (B). Sequential application of isoprenaline and diltiazem, the initial application of isoprenaline to a beating cardiac graft resulted in elevated chronotropic and ionotropic responses (red arrows). The successive application of a calcium channel blocker, diltiazem, to the same sample manifested in sustained negative chronotropic and ionotropic responses $(\mathbf{C})$. Representative calcium flux of spontaneously and rhythmically beating cells, that is, before the application of a sympathomimetic amine, $\beta_{2}$-agonist, clenbuterol (D), whereas, the application of clenbuterol to the same sample led to an elevated chronotropic effect (E). Sequential application of clenbuterol and diltiazem, the initial application of clenbuterol to a beating CCC cardiac graft displayed augmented chronotropic and ionotropic responses, while a subsequent application of diltiazem (blue arrows) progressively abrogated the contractility of cardiac graft (F). Calcium oscillations in response to an application of diltiazem alone to a spontaneously beating cardiac patch led to negative chronotropic and ionotropic responses, which eventually resulted in the arrest of cardiac patch contractility $(\mathbf{G})$. 
A

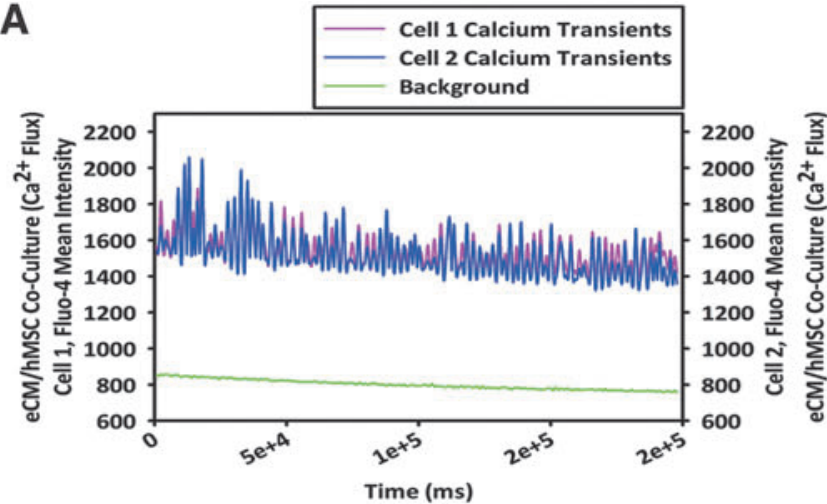

B

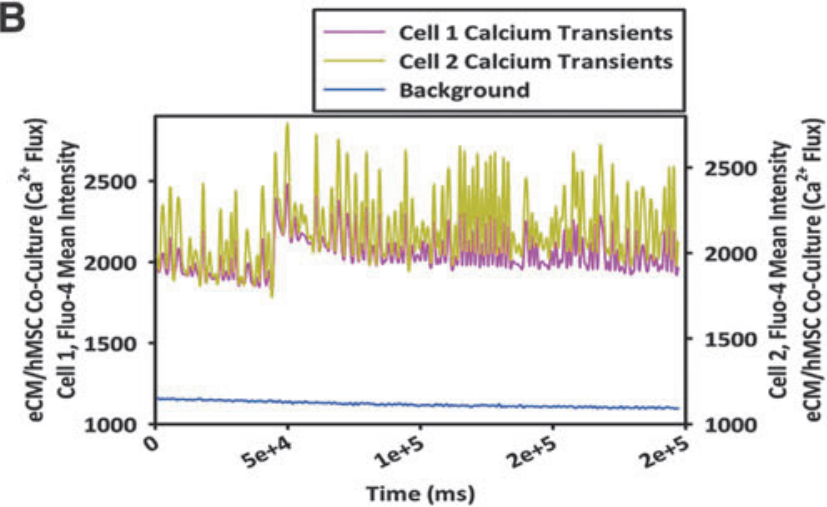

C

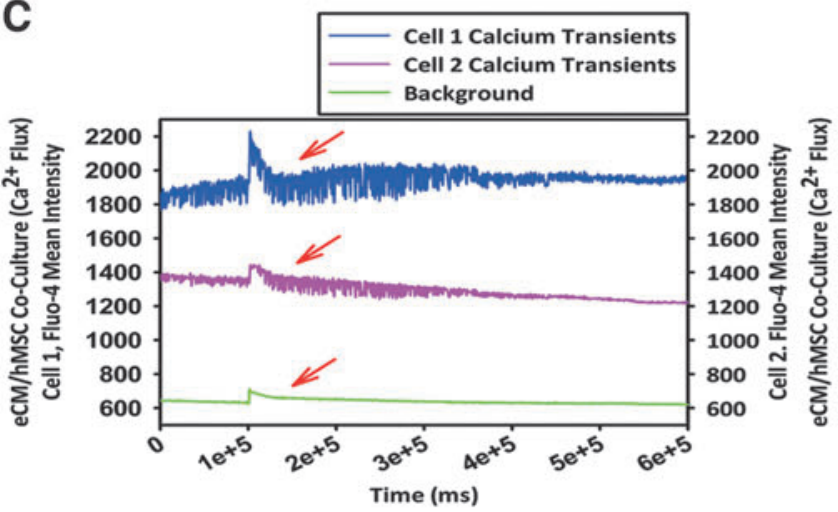

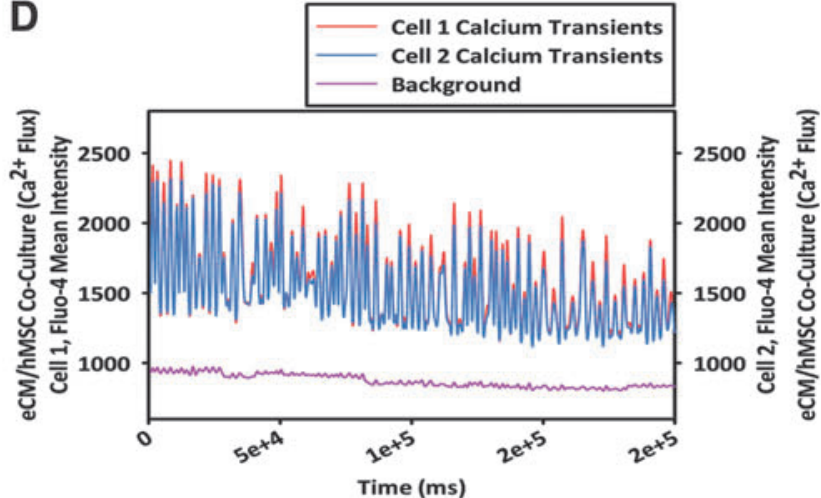

$\mathbf{E}$

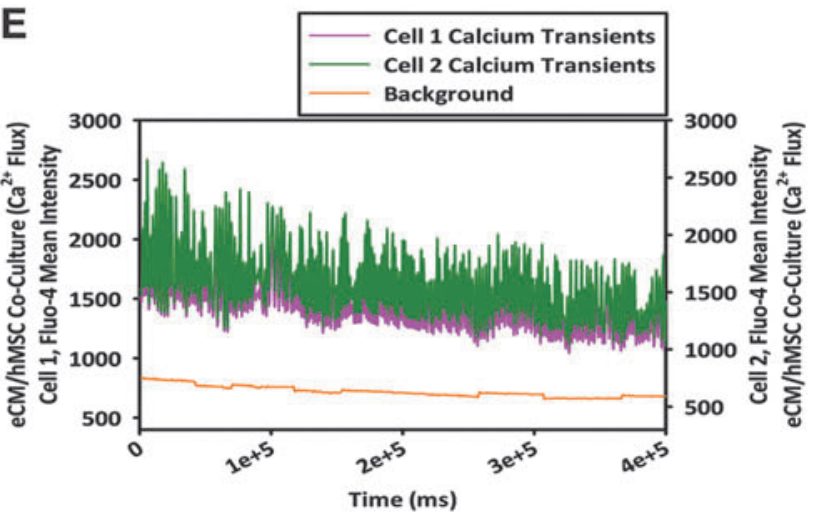

$\mathbf{F}$

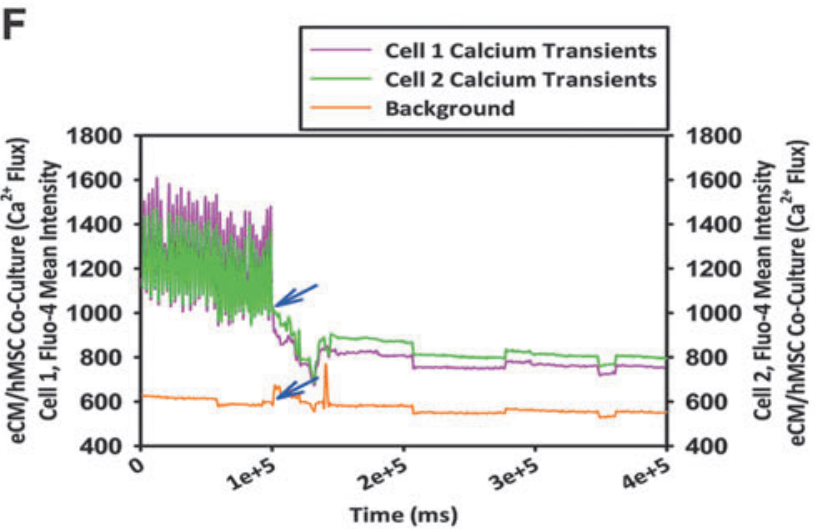

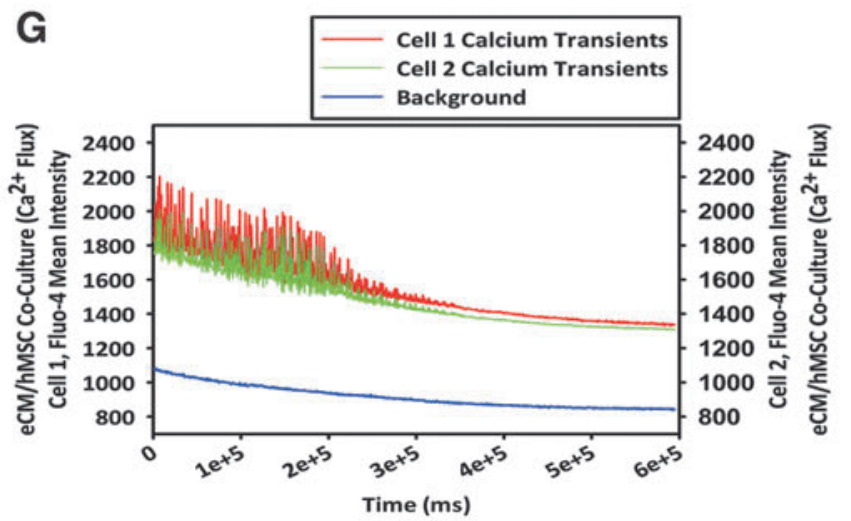



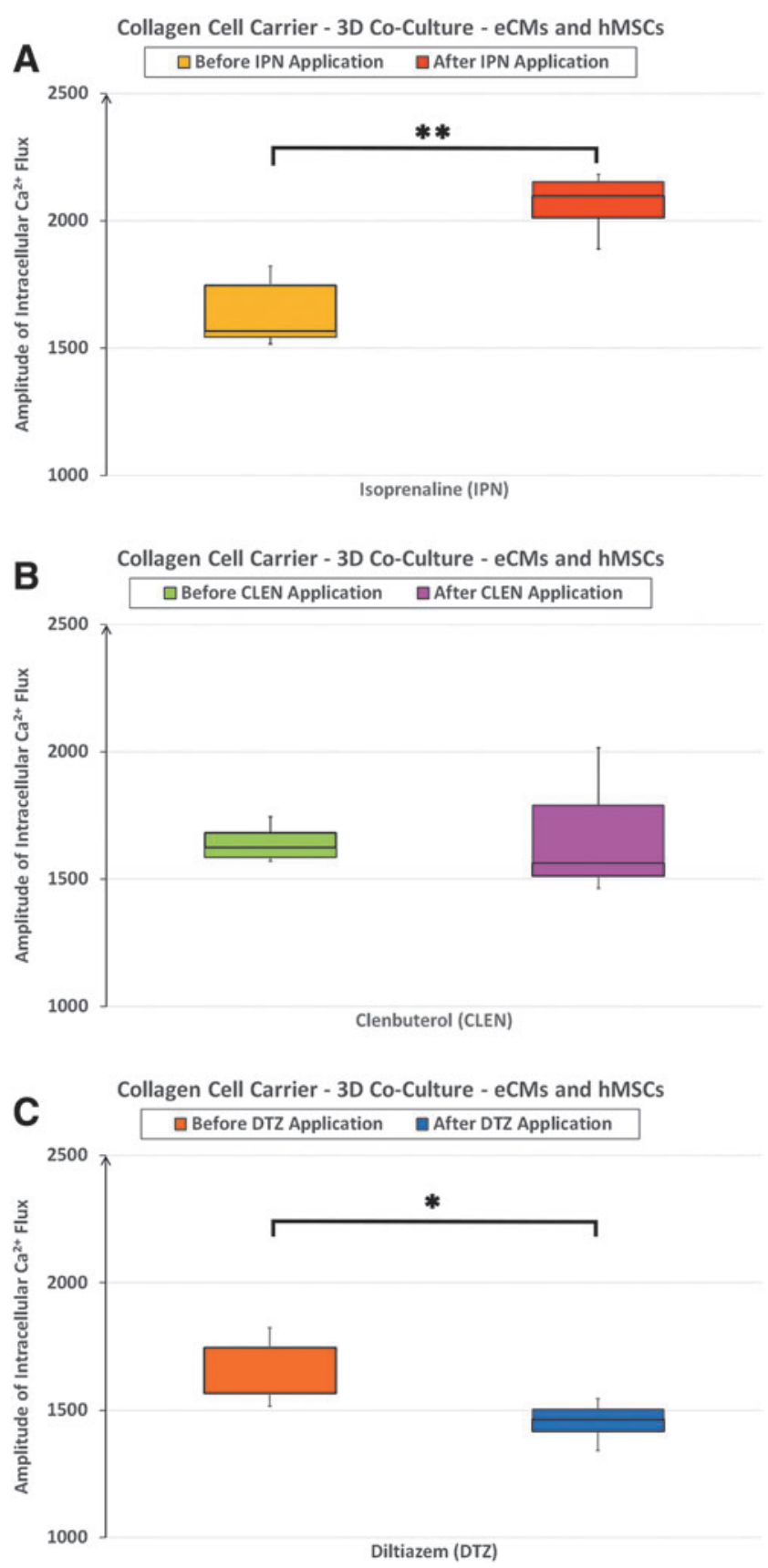

FIG. 16. Analysis of the influence of $\beta$-adrenoceptor activation and L-type calcium channel inhibition of vascularized cardiac muscle construct. The effect of $\beta$-adrenoceptor activation or L-type calcium channel inhibition of vascularized cardiac muscle constructs, and the corresponding quantitative changes in their magnitude of intracellular calcium oscillations: isoprenaline (A), clenbuterol (B), and diltiazem $(\mathbf{C})$. The number of replicates, referring to number of cells that were analyzed from five independent experiments $(n=10), * p<0.05 ; * * p<0.001$.

immune- and circulating blood-related cells. ${ }^{39}$ Despite the fact that cardiac myocytes constitute the majority of organ mass, cardiac myocytes constitute only twenty to thirty percent of the entire number of cells that are present in an adult heart. Even though cardiac myocyte mass is roughly twenty-five times that of endothelial cell mass, the smaller endothelial cells predominate myocytes by $\sim 3: 1{ }^{14}$ It has been shown that communication between these cell types in a network structure is vital for cardiac development, autoregulation, and adaptation. ${ }^{11,40}$

Besides, such cardiac endothelial-myocardial signaling may be even more critical during cellular stress, such as ischemic shock. ${ }^{39}$ Organized communication among the various cellular components of this functional syncytium is crucial not only with respect to normal cardiac metabolism, growth, contractile performance, and rhythmicity but also for adaptive and protective mechanisms to prevent against myocardial damage. ${ }^{39}$ Taken together, these facts suggest that one of the successful strategies for myocardial regeneration may therefore depend on establishing functional cardiac endothelium-myocyte communication and/or interactions.

So far, in regenerative medicine, cellular cardiomyoplasty is one of the promising options for treating ischemic heart diseases. Preliminary clinical studies have demonstrated that adult bone marrow-derived stem/precursor cells, in particular MSCs and/or EPCs, when preferentially transplanted into an acute myocardial infarction can have a certain degree of beneficial effects. ${ }^{41-52}$ Majority of these studies have shown that cell therapy reduced not only the size of an infarct but also concomitantly reduced ischemic burden, thereby improving cardiac contractile performance, that is, the ventricular function.

Multiple types of marrow-derived cell populations have experimentally been shown to have a certain degree of functional recovery of the heart after ischemia, and improve postinfarct cardiac repair by means of both neocardiomyogenesis and neo-angiogenesis, or alternatively, by secreting a cocktail of proangiogenic and antiapoptotic survival factors, thus promoting tissue repair in a paracrine manner. ${ }^{53}$ However, thus far, experimental studies addressing the capacity of transplanted bone marrow-derived stem cells to differentiate into cardiomyogenic lineage yielded conflicting results. ${ }^{43,54,55}$ The cell engraftment rate was shown to be rather low, reaching only $2-5 \%$ of the total number of transplanted cells. ${ }^{56}$ However, in this study, we have shown that in the presence of hCMVECs, the mechanically coupled hMSCs and eCMs exhibited synchronized calcium cycling and excitation-contraction coupling.

It has been shown that a variety of stem/precursor cell populations were able to contribute to neovascularization. Neovascularization can be facilitated by the physical incorporation of these progenitors into the developing new capillaries $^{57}$ or simply by perivascular amassing of cells. Moreover, most of the progenitors, although not all types, may release a cocktail of growth factors, which may promote angiogenesis by acting on mature endothelium. ${ }^{58}$ Nonetheless, the extent to which progenitors contribute to vasculogenesis by literally getting integrated into newly formed vasculature versus acting through secreted factors may plausibly depend on the microenvironment with which the cells are exposed, and may, in part, depend on the nature of the applied cell type. This could explain the large discrepancies in endothelial cell incorporation detected in different experimental studies, currently.

Nonetheless, in this study, we have shown that hMSCs in the presence of hCMVECs can unequivocally contribute to the development of tunica media, by differentiating in this milieu into $\alpha$-SMA-positive smooth muscle cells, and are 
able to spontaneously integrate and wrap around the endothelium-lined tubular elements. Furthermore, it goes without notice that our 3D model of vascularized cardiac tissue can be utilized, for example, to gain a deeper insight and a clearer understanding of various competing hypotheses concerning the development of neo-vascular structures in vitro, during repair and/or regeneration.

Failure of cell-based therapy for ischemic heart disease in regenerative medicine, presently, relies on tissue-engineered cardiac grafts; tissue engineering holds great promises to enhance cardiac function by improving or restoring both electrical and mechanical activation of damaged myocardial regions. At present, an important goal in cardiac therapy is the quest to improve cell engraftment, survival, and functional integration of cells at the site of implantation, ultimately, to recreate normal myocardial architecture and anisotropy. 59

Until now, cell therapy is at its infancy, and various questions remain to be answered. One of the pressing questions in basic cardiovascular sciences is to elucidate the various molecular mechanisms that are governing by which stem/precursor cells achieve a functional improvement, which is in general difficult to be tested and/or achieved in the clinical scenario. As a result, this necessitates the prompt development of an appropriate in vitro $3 \mathrm{D}$ model of cardiomyogenesis and prompts us the development of a $3 \mathrm{D}$ vascularized cardiac muscle construct for tissue engineering and personalized medicine purposes, especially, by means of a hybrid/combinatorial approach, that is, using a combination of biomaterial (CCCs), cardiomyocytes (eCMs), the putative somatic stem cells (hMSCs), and supporting noncardiomyocytes, such as the primary endothelial cells (hCMVECs).

Previously, Zimmermann et al., ${ }^{60}$ have shown that it has been possible to generate an in vitro $3 \mathrm{D}$ cardiac muscle construct consisting of capillary-like structures by employing tissue engineering principles. The construct consisted of rat biopsy-derived neonatal cardiac myocytes along with other types of cells. The biopsy-derived cells were admixed with extracellular matrix materials, the type I collagen, and other matrix factors, casted in circular molds, and subjected to phasic mechanical stretching. The reconstituted cardiac construct displayed not only the hallmarks of differentiating myocardium but also revealed very occasional PECAM1positive vessel-like structures. ${ }^{60}$

Similarly, Caspi et al. ${ }^{61}$ have demonstrated that it was feasible to recreate in vitro the formation of vascularized tissue-engineered cardiac tissue, which composed of human pluripotent stem cell-derived eCMs and endothelial cells, as well as mouse embryo-derived embryonic fibroblasts. When eCMs and endothelial cells were cocultured together on a biodegradable porous sponge (composed of poly-L-lactic acid [PLLA] and poly-lactic glycolic acid [PLGA], 50\% PLLA/50\% PLGA), it did not result in endothelial cell reorganization or self-assembly, or tube formation. However, when the coculture was admixed with fibroblasts, the resulting tri-cell coculture generated a vascularized construct consisting of a noticeable number of capillaries that were stabilized by fibroblast-derived mural cells. Thus, interaction among various cell types not only supported microvessel formation but also enhanced the proliferation and/or differentiation of primitive cardiac myocytes. ${ }^{61}$

Unlike these previous reports, ${ }^{60,61}$ our study indicated that the mere presence of hMSCs evidently enhanced the angiogenic potential of hCMVECs in this milieu, and this could possibly be attributed to the proangiogenic and prosurvival properties of hMSCs. ${ }^{53}$ Besides, unlike fibroblasts, the other potential advantage of utilizing hMSCs is that reciprocal induction and differentiation of hMSCs and hCMVECs remarkably promoted the generation of in situ de novo large-caliber vessels in this microenvironmental niche. Finally, hMSCs have been reported to be immunomodulatory and immunotolerogenic both in vitro and in vivo. ${ }^{62}$ Collectively, these observations would suggest that autologous or allogeneic hMSCs can serve as a potential cellular source for vascular tissue engineering purposes. ${ }^{36}$

The cell carrier, CCC, is a unique, thin, and planar collagen scaffold. CCCs are engineered from pure bovine type I collagen fibers, and are robust and well suited for growth and/or differentiation of various types of adherent primary cells, stem cells, and transformed cell lines. It demonstrates a low material thickness of around $40 \mu \mathrm{m}$ coupled with a high mechanical strength, on tensile test analysis. ${ }^{24}$ In the field of regenerative medicine, the high biocompatibility and high mechanical strength make these collagen membranes ideal for transplantation experiments, as they permit easy maneuverability and portability of sterile and intact cell scaffold complex. ${ }^{24}$

The other advantages of CCC are its in vivo-like collagen topography (since it contains noncrosslinked native collagen fibers), which permits incorporating various combinations of additional growth factors and/or matrix molecules. Most importantly, even though by nature CCCs are compact and nonporous, they are still permeable to soluble factors. Being conducive and allowing the directed differentiation of stem/ progenitor cells, it represents an excellent scaffold for complex tissues and tissue engineering purposes. The other advantage is that CCCs are scalable, that is, its dimensions can be tailor-made as situation warrants.

Our data have provided compelling evidence that presence of preexisting microvessels, in fact, is able to augment and maintain an in vivo-like phenotype of ventricularderived eCMs. Besides, we have shown the continuum of progressive maturation and differentiation of these ventricular eCMs, from embryonic to neonatal-type of phenotype. Since functional integration of transplanted cells within host cardiac tissue is of paramount import, we have shown that hMSCs are able to be coupled with juxtaposed cardiomyocytes, and are able to display calcium oscillations almost at the same amplitude and frequency as the neighboring myocytes. Not only that, we have also shown that hMSCs are able to align with eCMs to restore tissue architecture, and thereby an anisotropic conduction is persevered. Finally, we have demonstrated the presence of gap junction proteins, including connexin 43 , which is localized at the intercalated discs, hence decreasing the electrical heterogeneity as well as preventing undue arrhythmias.

In addition, we have provided compelling evidence that hMSCs are not only mechanically coupled but also are able to form a functional syncytium, and able to function in synchrony with the eCMs. The significance of addressing this issue, that is, the electrical homogeneity, is highlighted by the fact that in human heart failures, despite the presence of electrical couplings in all of the myocardial cells, these cells do not always produce a synchronized contraction. However, in our study, hMSCs are able to achieve functional 
competence and not mere electrical or mechanical integration, thus hMSCs are not only electromechanically similar to each other but also functionally resemble that of eCMs.

Considering the interplay of cardiac endothelial-myocytes in cardiac development and the presence of microvascular endothelial cells (hCMVECs) and eCMs, we have demonstrated that hMSC-derived smooth muscle cells (positive for $\alpha-S M A$ antigen) explicitly contributed to the evolution of tunica media of the large-caliber vessels, which is in addition to the demonstration of hMSC-derived neo-myocytes in this cocultured environment. However, hMSCs in these environmental niches do not depict the ultrastructural characteristics of mature and terminally differentiated adult cardiac myocytes, such as formation of subsarcolemmal and/or intermyofibrillar array of mitochondria. Consequently, additional strategies may need to be employed to achieve the process of terminal differentiation of myocytes. Therefore, terminal differentiation of hMSCs/eCMs can be accomplished by means of mechanical load since it has been shown that mechanical forces contribute to the process of differentiation and morphogenesis from the level of individual cells to whole organism patterning. ${ }^{63}$

It is immediately obvious to us because CCCs are robust and allow for flexibility and versatility when it comes to mechanical forces, and is more amenable to various mechanical stimuli, such as stretching and/or hydrodynamic forces. Accordingly, it is an ideal model system to test further the effects of cell-cell and cell-mechanotransduction on the maturation of the generated vascularized cardiac muscle construct.

Previous studies have suggested that using hypoxiapreconditioned (HP) MSCs enhances the cell survival of implanted cells and angiogenesis, ${ }^{64-66}$ and further proceeded to show that HP increased expression of prosurvival and proangiogenic factors (e.g., hypoxia-inducible factor1A [HIF1A]). In addition, transplantation of hypoxic versus normoxic stem cells after myocardial infarction resulted in an increase in angiogenesis as well as enhanced functional benefits. Therefore, it stands to reason that using a similar approach with our cardiac construct would more likely produce even greater vasculogenesis and more mature vessels.

Our results clearly illustrate that it is possible to accomplish in vitro the development of a mammalian myocardial tissue, simply by employing the evolving concept of tissue engineering-based modeling of morphogenesis. "Modeling morphogenesis, where it seeks to persuade simple collection of cells to undergo self-assembly or react to an unnatural environment without the presence of a preexisting template.",67 The tissue engineering approaches have provided the best way to verify a very fundamental and important hypothesis, that is, the behavior of a particular cell depends not on its past history (predetermination/memory), but depends on here and now, that is, existence at this very moment. ${ }^{67}$ This means that a cell's history can naturally determine its state of differentiation, and consequently, it relies on which morphogenetic machines are existing in it. However, the morphogenetic behavior of those cellular machines is supposed to depend on the physicochemical influences present, such as physical, chemical, mechanical, and electrical factors, and not on a long-term memory of previous events. ${ }^{67}$ In a nutshell, cells are capable of adaptive self-organization based on present circumstances and constraints.

Typically, hMSC-derived colonies are clonal or nearly clonal. Moreover, colonies of hMSCs derived from several cells may comprise of a number of clones, each capable of differentiating into specific lineages. ${ }^{21}$ Consequently, to assess and delineate the exact fate and contribution of hMSCs toward various cellular lineages, such as endothelial, smooth muscle, and striated muscle, in the cardiac patch, it is imperative to utilize clonally expanded hMSCs to arrive at more meaningful conclusions.

Finally, the vital issue for realistic clinical application is whether these scaffolds with preformed microvessels can survive implantation into tissue defects, and subsequently be able to anastomose with the host vasculature. Further experiments would be needed to address this critical issue; thus, in vivo implantation experiments are underway to ascertain the suitability of the prevascularized CCC cardiac grafts on various types of ischemic and nonischemic cardiac lesions, especially using constructs that are composed of a combination of sex-mismatched and/or genetically or fluorescently marked cells.

\section{Conclusions}

In this study, we report the creation of a reproducible and prototypical in vitro 3D prevascularized cardiac tissue, which encompasses all the indispensable characteristics of a tissue-engineered cardiac graft, such as consistent and synchronized spontaneous contractility, stable and responsive electrophysiological properties, vascularization, and a somatic stem cell source, to address the most chronic problem that is facing cardiac therapy as of today, that is, the repair and/or regeneration of damaged myocardium. In addition, we have demonstrated that it is feasible to recreate in vitro a 3D model of mammalian cardiomyogenesis that has been long awaited.

Moreover, this 3D vascularized cardiac tissue can be utilized to address various outstanding questions that linger in the highly controversial field of adult stem cell-based cardiac regeneration, and allows us to dissect various molecular mechanisms that are underpinning the orderly cellular differentiation and tissue morphogenesis, which are probably difficult to be examined and/or accomplished in the clinical scenario. Eventually, the outcome from this study may translate to human personalized medicine by rendering a targeted approach to regulate the maintenance (physiological) or reparative (pathological) process of myocardium and, in turn, can lead to functional enhancement of a failing heart.

\section{Authorship}

We confirm that M.T.V., J.W.F., J.D.P., J.M.D., and R.L.P. meet the stipulated criteria for authorship of this original article based on the recommendation of the ICMJE.

\section{Acknowledgments}

The authors thank Ms. Jiang Li for her enthusiastic and excellent technical support. "This work was supported by an award from the American Heart Association."-National Scientist Development Grant (11SDG5280022) for M.T.V. 


\section{Disclosure Statement}

No competing financial interests exist.

\section{References}

1. Soonpaa, M.H., and Field, L.J. Survey of studies examining mammalian cardiomyocyte DNA synthesis. Circ Res 83, $15,1998$.

2. Leor, J., Amsalem, Y., and Cohen, S. Cells, scaffolds, and molecules for myocardial tissue engineering. Pharmacol Ther 105, 151, 2005.

3. Meyer, U., Meyer, T., Handschel, J., and Wiesmann, H.P. Fundamentals of Tissue Engineering and Regenerative Medicine. Berlin, Heidelberg: Springer, 2009.

4. Bursac, N., Papadaki, M., Cohen, R.J., Schoen, F.J., Eisenberg, S.R., Carrier, R., Vunjak-Novakovic, G., and Freed, L.E. Cardiac muscle tissue engineering: toward an in vitro model for electrophysiological studies. Am J Physiol 277, H433, 1999.

5. Zimmermann, W.H., Fink, C., Kralisch, D., Remmers, U., Weil, J., and Eschenhagen, T. Three-dimensional engineered heart tissue from neonatal rat cardiac myocytes. Biotechnol Bioeng 68, 106, 2000 .

6. Papadaki, M., Bursac, N., Langer, R., Merok, J., VunjakNovakovic, G., and Freed, L.E. Tissue engineering of functional cardiac muscle: molecular, structural, and electrophysiological studies. Am J Physiol Heart Circ Physiol 280, H168, 2001.

7. Rivron, N.C., Liu, J.J., Rouwkema, J., de Boer, J., and van Blitterswijk, C.A. Engineering vascularised tissues in vitro. Eur Cell Mater 15, 27, 2008.

8. Ennett, A.B., and Mooney, D.J. Tissue engineering strategies for in vivo neovascularisation. Expert Opin Biol Ther 2, 805, 2002.

9. Jain, R.K. Molecular regulation of vessel maturation. Nat Med 9, 685, 2003.

10. Levenberg, S., Rouwkema, J., Macdonald, M., Garfein, E.S., Kohane, D.S., Darland, D.C., Marini, R., van Blitterswijk, C.A., Mulligan, R.C., D’Amore, P.A., and Langer, R. Engineering vascularized skeletal muscle tissue. Nat Biotechnol 23, 879, 2005.

11. Brutsaert, D.L. Cardiac endothelial-myocardial signaling: its role in cardiac growth, contractile performance, and rhythmicity. Physiol Rev 83, 59, 2003.

12. Cleaver, O., and Melton, D.A. Endothelial signaling during development. Nat Med 9, 661, 2003.

13. Hsieh, P.C., Davis, M.E., Lisowski, L.K., and Lee, R.T. Endothelial-cardiomyocyte interactions in cardiac development and repair. Annu Rev Physiol 68, 51, 2006.

14. Brutsaert, D.L., Fransen, P., Andries, L.J., De Keulenaer, G.W., and Sys, S.U. Cardiac endothelium and myocardial function. Cardiovasc Res 38, 281, 1998.

15. Narmoneva, D.A., Vukmirovic, R., Davis, M.E., Kamm, R.D., and Lee, R.T. Endothelial cells promote cardiac myocyte survival and spatial reorganization: implications for cardiac regeneration. Circulation 110, 962, 2004.

16. Nishida, M., Springhorn, J.P., Kelly, R.A., and Smith, T.W. Cell-cell signaling between adult rat ventricular myocytes and cardiac microvascular endothelial cells in heterotypic primary culture. J Clin Invest 91, 1934, 1993.

17. Kuramochi, Y., Cote, G.M., Guo, X., Lebrasseur, N.K., Cui, L., Liao, R., and Sawyer, D.B. Cardiac endothelial cells regulate reactive oxygen species-induced cardiomyocyte apoptosis through neuregulin-1beta/erbB4 signaling. J Biol Chem 279, 51141, 2004.
18. Ramaciotti, C., Sharkey, A., McClellan, G., and Winegrad, S. Endothelial cells regulate cardiac contractility. Proc Natl Acad Sci U S A 89, 4033, 1992.

19. Simpson, D.G., Terracio, L., Terracio, M., Price, R.L., Turner, D.C., and Borg, T.K. Modulation of cardiac myocyte phenotype in vitro by the composition and orientation of the extracellular matrix. J Cell Physiol 161, 89, 1994.

20. Valarmathi, M.T., Fuseler, J.W., Goodwin, R.L., Davis, J.M., and Potts, J.D. The mechanical coupling of adult marrow stromal stem cells during cardiac regeneration assessed in a 2-D co-culture model. Biomaterials 32, 2834, 2011.

21. Valarmathi, M.T., Yost, M.J., Goodwin, R.L., and Potts, J.D. A three-dimensional tubular scaffold that modulates the osteogenic and vasculogenic differentiation of rat bone marrow stromal cells. Tissue Eng Part A 14, 491, 2008.

22. Fuseler, J.W., and Valarmathi, M.T. Modulation of the migration and differentiation potential of adult bone marrow stromal stem cells by nitric oxide. Biomaterials 33, 1032, 2012.

23. Valarmathi, M.T., Goodwin, R.L., Fuseler, J.W., Davis, J.M., Yost, M.J., and Potts, J.D. A 3-D cardiac muscle construct for exploring adult marrow stem cell based myocardial regeneration. Biomaterials 31, 3185, 2010.

24. Schmidt, T., Stachon, S., Mack, A., Rohde, M., and Just, L. Evaluation of a thin and mechanically stable collagen cell carrier. Tissue Eng Part C Methods 17, 1161, 2011.

25. Voyta, J.C., Via, D.P., Butterfield, C.E., and Zetter, B.R. Identification and isolation of endothelial cells based on their increased uptake of acetylated-low density lipoprotein. J Cell Biol 99, 2034, 1984.

26. Rasband, W. ImageJ. Bethesda, MD: National Institute of Health. https://imagej.nih.gov/ij/ (last accessed March 2017).

27. Gholobova, D., Decroix, L., Van Muylder, V., Desender, L., Gerard, M., Carpentier, G., Vandenburgh, H., and Thorrez, L. Endothelial network formation within human tissueengineered skeletal muscle. Tissue Eng Part A 21, 2548, 2015.

28. Carpentier, G. Gilles Carpentier research web site: computer image analysis. Universite Paris Est-Creteil Val-de-Marne, France: Angiogenesis Analyzer for ImageJ, 2012.

29. Hanaichi, T., Sato, T., Iwamoto, T., Malavasi-Yamashiro, J., Hoshino, M., and Mizuno, N. A stable lead by modification of Sato's method. Journal of electron microscopy 35, 304, 1986.

30. Rosen, S., and Skaletsky, H.J. Primer3 on the www for general users and for biologist programmers. In: Krawetz, S., and Mlsener, S., eds. Methods in Molecular Biology. Totowa, NJ: Human Press, 2000, Pp. 365.

31. Valarmathi, M.T., Yost, M.J., Goodwin, R.L., and Potts, J.D. The influence of proepicardial cells on the osteogenic potential of marrow stromal cells in a three-dimensional tubular scaffold. Biomaterials 29, 2203, 2008.

32. Bustin, S.A., Benes, V., Garson, J.A., Hellemans, J., Huggett, J., Kubista, M., Mueller, R., Nolan, T., Pfaffl, M.W., Shipley, G.L., Vandesompele, J., and Wittwer, C.T. The MIQE guidelines: minimum information for publication of quantitative real-time PCR experiments. Clin Chem 55, 611, 2009.

33. Willems, E., Leyns, L., and Vandesompele, J. Standardization of real-time PCR gene expression data from independent biological replicates. Anal Biochem 379, 127, 2008.

34. Pfaffl, M.W., Horgan, G.W., and Dempfle, L. Relative expression software tool (REST) for group-wise compari- 
son and statistical analysis of relative expression results in real-time PCR. Nucleic Acids Res 30, e36, 2002.

35. Dominici, M., Le Blanc, K., Mueller, I., Slaper-Cortenbach, I., Marini, F., Krause, D., Deans, R., Keating, A., Prockop, D., and Horwitz, E. Minimal criteria for defining multipotent mesenchymal stromal cells. The International Society for Cellular Therapy position statement. Cytotherapy 8, 315, 2006.

36. Valarmathi, M.T., Davis, J.M., Yost, M.J., Goodwin, R.L., and Potts, J.D. A three-dimensional model of vasculogenesis. Biomaterials 30, 1098, 2009.

37. Valarmathi, M.T., Fuseler, J.W., Davis, J.M., and Price, R.L. A novel human tissue-engineered 3-D functional vascularized cardiac muscle construct. Front Cell Dev Biol $\mathbf{5}, 2,2017$.

38. Tirziu, D., and Simons, M. Endothelium as master regulator of organ development and growth. Vascul Pharmacol 50, 1, 2009.

39. Leucker, T.M., and Jones, S.P. Endothelial dysfunction as a nexus for endothelial cell-cardiomyocyte miscommunication. Front Physiol 5, 328, 2014.

40. Tirziu, D., Giordano, F.J., and Simons, M. Cell communications in the heart. Circulation 122, 928, 2010.

41. Fuchs, E., and Segre, J.A. Stem cells: a new lease on life. Cell 100, 143, 2000.

42. Jackson, K.A., Majka, S.M., Wang, H., Pocius, J., Hartley, C.J., Majesky, M.W., Entman, M.L., Michael, L.H., Hirschi, K.K., and Goodell, M.A. Regeneration of ischemic cardiac muscle and vascular endothelium by adult stem cells. J Clin Invest 107, 1395, 2001.

43. Orlic, D., Kajstura, J., Chimenti, S., Jakoniuk, I., Anderson, S.M., Li, B., Pickel, J., McKay, R., Nadal-Ginard, B., Bodine, D.M., Leri, A., and Anversa, P. Bone marrow cells regenerate infarcted myocardium. Nature 410, 701, 2001.

44. Makino, S., Fukuda, K., Miyoshi, S., Konishi, F., Kodama, H., Pan, J., Sano, M., Takahashi, T., Hori, S., Abe, H., Hata, J., Umezawa, A., and Ogawa, S. Cardiomyocytes can be generated from marrow stromal cells in vitro. J Clin Invest 103, 697, 1999.

45. Liechty, K.W., MacKenzie, T.C., Shaaban, A.F., Radu, A., Moseley, A.M., Deans, R., Marshak, D.R., and Flake, A.W. Human mesenchymal stem cells engraft and demonstrate site-specific differentiation after in utero transplantation in sheep. Nat Med 6, 1282, 2000.

46. Shake, J.G., Gruber, P.J., Baumgartner, W.A., Senechal, G., Meyers, J., Redmond, J.M., Pittenger, M.F., and Martin, B.J. Mesenchymal stem cell implantation in a swine myocardial infarct model: engraftment and functional effects. Ann Thorac Surg 73, 1919, 2002.

47. Toma, C., Pittenger, M.F., Cahill, K.S., Byrne, B.J., and Kessler, P.D. Human mesenchymal stem cells differentiate to a cardiomyocyte phenotype in the adult murine heart. Circulation 105, 93, 2002.

48. Chen, S.L., Fang, W.W., Ye, F., Liu, Y.H., Qian, J., Shan, S.J., Zhang, J.J., Chunhua, R.Z., Liao, L.M., Lin, S., and Sun, J.P. Effect on left ventricular function of intracoronary transplantation of autologous bone marrow mesenchymal stem cell in patients with acute myocardial infarction. Am J Cardiol 94, 92, 2004.

49. Min, J.Y., Sullivan, M.F., Yang, Y., Zhang, J.P., Converso, K.L., Morgan, J.P., and Xiao, Y.F. Significant improvement of heart function by cotransplantation of human mesenchymal stem cells and fetal cardiomyocytes in postinfarcted pigs. Ann Thorac Surg 74, 1568, 2002.
50. Janssens, S., Dubois, C., Bogaert, J., Theunissen, K., Deroose, C., Desmet, W., Kalantzi, M., Herbots, L., Sinnaeve, P., Dens, J., Maertens, J., Rademakers, F., Dymarkowski, S., Gheysens, O., Van Cleemput, J., Bormans, G., Nuyts, J., Belmans, A., Mortelmans, L., Boogaerts, M., and Van de Werf, F. Autologous bone marrow-derived stem-cell transfer in patients with ST-segment elevation myocardial infarction: double-blind, randomized controlled trial. Lancet 367, 113, 2006.

51. Cleland, J.G., Freemantle, N., Coletta, A.P., and Clark, A.L. Clinical trials update from the American Heart Association: REPAIR-AMI, ASTAMI, JELIS, MEGA, REVIVE-II, SURVIVE, and PROACTIVE. Eur J Heart Fail 8, 105, 2006.

52. Zimmet, J.M., and Hare, J.M. Emerging role for bone marrow derived mesenchymal stem cells in myocardial regenerative therapy. Basic Res Cardiol 100, 471, 2005.

53. Dimmeler, S., Burchfield, J., and Zeiher, A.M. Cell-based therapy of myocardial infarction. Arterioscler Thromb Vasc Biol 28, 208, 2008.

54. Murry, C.E., Soonpaa, M.H., Reinecke, H., Nakajima, H., Nakajima, H.O., Rubart, M., Pasumarthi, K.B., Virag, J.I., Bartelmez, S.H., Poppa, V., Bradford, G., Dowell, J.D., Williams, D.A., and Field, L.J. Haematopoietic stem cells do not transdifferentiate into cardiac myocytes in myocardial infarcts. Nature 428, 664, 2004.

55. Anversa, P., Leri, A., Rota, M., Hosoda, T., Bearzi, C., Urbanek, K., Kajstura, J., and Bolli, R. Concise review: stem cells, myocardial regeneration, and methodological artifacts. Stem Cells 25, 589, 2007.

56. Grauss, R.W., van Tuyn, J., Steendijk, P., Winter, E.M., Pijnappels, D.A., Hogers, B., Gittenberger-De Groot, A.C., van der Geest, R., van der Laarse, A., de Vries, A.A., Schalij, M.J., and Atsma, D.E. Forced myocardin expression enhances the therapeutic effect of human mesenchymal stem cells after transplantation in ischemic mouse hearts. Stem Cells 26, 1083, 2008.

57. Kawamoto, A., Tkebuchava, T., Yamaguchi, J., Nishimura, H., Yoon, Y.S., Milliken, C., Uchida, S., Masuo, O., Iwaguro, H., Ma, H., Hanley, A., Silver, M., Kearney, M., Losordo, D.W., Isner, J.M., and Asahara, T. Intramyocardial transplantation of autologous endothelial progenitor cells for therapeutic neovascularization of myocardial ischemia. Circulation 107, 461, 2003.

58. Urbich, C., Aicher, A., Heeschen, C., Dernbach, E., Hofmann, W.K., Zeiher, A.M., and Dimmeler, S. Soluble factors released by endothelial progenitor cells promote migration of endothelial cells and cardiac resident progenitor cells. J Mol Cell Cardiol 39, 733, 2005.

59. Pijnappels, D.A., Gregoire, S., and Wu, S.M. The integrative aspects of cardiac physiology and their implications for cell-based therapy. Ann N Y Acad Sci 1188, 7, 2010.

60. Zimmermann, W.H., Schneiderbanger, K., Schubert, P., Didie, M., Munzel, F., Heubach, J.F., Kostin, S., Neuhuber, W.L., and Eschenhagen, T. Tissue engineering of a differentiated cardiac muscle construct. Circ Res 90, 223, 2002.

61. Caspi, O., Lesman, A., Basevitch, Y., Gepstein, A., Arbel, G., Habib, I.H., Gepstein, L., and Levenberg, S. Tissue engineering of vascularized cardiac muscle from human embryonic stem cells. Circ Res 100, 263, 2007.

62. Aggarwal, S., and Pittenger, M.F. Human mesenchymal stem cells modulate allogeneic immune cell responses. Blood 105, 1815, 2005. 
63. Shav, D., and Einav, S. The effect of mechanical loads in the differentiation of precursor cells into mature cells. Ann N Y Acad Sci 1188, 25, 2010.

64. Hu, X., Yu, S.P., Fraser, J.L., Lu, Z., Ogle, M.E., Wang, J.A., and Wei, L. Transplantation of hypoxia-preconditioned mesenchymal stem cells improves infarcted heart function via enhanced survival of implanted cells and angiogenesis. J Thorac Cardiovasc Surg 135, 799, 2008.

65. Hamano, K., Li, T.S., Kobayashi, T., Kobayashi, S., Matsuzaki, M., and Esato, K. Angiogenesis induced by the implantation of self-bone marrow cells: a new material for therapeutic angiogenesis. Cell Transplant 9, 439, 2000.

66. Silva, G.V., Litovsky, S., Assad, J.A., Sousa, A.L., Martin, B.J., Vela, D., Coulter, S.C., Lin, J., Ober, J., Vaughn, W.K., Branco, R.V., Oliveira, E.M., He, R., Geng, Y.J., Willerson, J.T., and Perin, E.C. Mesenchymal stem cells differentiate into an endothelial phenotype, enhance vascular density, and improve heart function in a canine chronic ischemia model. Circulation 111, 150, 2005.
67. Davies, J.A. Mechanisms of Morphogenesis, 2nd ed. Oxford, UK: Elsevier, Academic Press, 2013.

Address correspondence to: Mani T. Valarmathi, $M D, P h D$ Department of Comparative Biosciences College of Veterinary Medicine University of Illinois at Urbana-Champaign 3840 Veterinary Medicine Basic Sciences Building, MC-002 2001 South Lincoln Avenue Urbana, IL 61802

E-mail: tmv@illinois.edu; valarmathi64@hotmail.com

Received: December 7, 2016 Accepted: April 13, 2017

Online Publication Date: May 25, 2017 